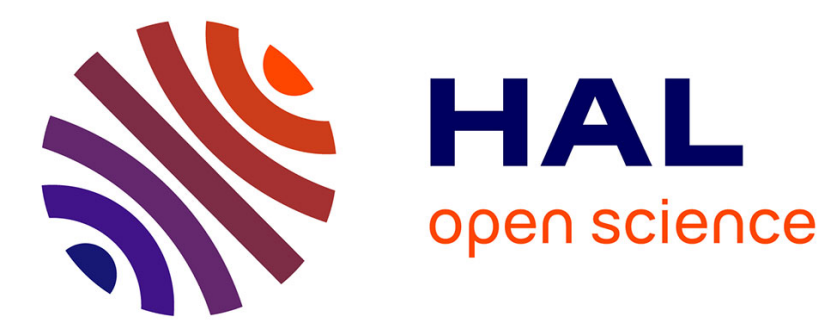

\title{
Adaptive monitoring system for e-health smart homes
}

Haider Mshali, Tayeb Lemlouma, Damien Magoni

\section{To cite this version:}

Haider Mshali, Tayeb Lemlouma, Damien Magoni. Adaptive monitoring system for e-health smart homes. Pervasive and Mobile Computing, 2018, 43, pp.1 - 19. 10.1016/j.pmcj.2017.11.001 . hal01646731

\section{HAL Id: hal-01646731 \\ https://hal.science/hal-01646731}

Submitted on 23 Nov 2017

HAL is a multi-disciplinary open access archive for the deposit and dissemination of scientific research documents, whether they are published or not. The documents may come from teaching and research institutions in France or abroad, or from public or private research centers.
L'archive ouverte pluridisciplinaire HAL, est destinée au dépôt et à la diffusion de documents scientifiques de niveau recherche, publiés ou non, émanant des établissements d'enseignement et de recherche français ou étrangers, des laboratoires publics ou privés. 


\title{
Adaptive Monitoring System for e-Health Smart Homes
}

\author{
Haider Mshali $^{\mathrm{a}}$, Tayeb Lemlouma ${ }^{\mathrm{b}, *}$, Damien Magoni $^{\mathrm{a}}$ \\ ${ }^{a}$ University of Bordeaux, LaBRI, 351 Cours de la Liberation, 33405 Talence, France \\ ${ }^{b}$ University of Rennes 1, IRISA, Rue Edouard Branly, 22302 Lannion, France
}

\begin{abstract}
Existing e-health monitoring systems mainly operate in isolation from the requirements of modern healthcare institutions. They do not include optimized techniques which learn the patient's behavior for predicting future important changes. We propose a new context-aware e-health monitoring system targeted at the elderly and isolated persons living alone. It monitors daily living activities and evaluates dependency based on geriatric scales used by health professionals. Its adaptive framework collects only relevant contextual data for evaluating health status. By monitoring the achievement of daily activities, the system learns the behavior of the monitored person. It is then able to detect risky behavioral changes by using our novel forecasting approach based on the extension of the Grey model $\operatorname{GM}(1,1)$. In order to evaluate our system, we use a Markovian model built for generating long term realistic scenarios. By simulation, we compare the performances of our system to traditional monitoring approaches with various synthetically generated scenarios and profiles. Results show that with minimal sensing and data collection, our system accurately evaluates a person's dependency, predicts its health condition, and detects abnormal situations while preserving system resources.
\end{abstract}

Keywords: abnormal behavior detection, adaptive context-aware monitoring, dependency, e-health, health smart home, health state prediction.

\section{Introduction}

The ratio of aging population has risen significantly in the past few years [1. The progressive decline in physical and cognitive skills prevents elderly people from living independently and from performing basic instrumental activities of daily living (IADL). A potential solution to this issue is to develop e-health monitoring systems (HMS) implemented at home, leading to the creation of health smart homes (HSH). HSH can monitor patients' activities and enable healthcare services at home especially for persons with chronic diseases. They can thus delay their placement in institutions such as nursing homes and hospitals.

Traditional monitoring systems tend to manage all sensed data with unconditional processing. Most of them adopt a continuous monitoring strategy that negatively affect resource usage and relevance of decisions. Such long-term monitoring consumes storage, uses energy for multiple sensors and sinks, increases computational costs required to analyze data, and increases network usage leading to transmission failures. Handling huge amounts of data can also impair the system in triggering relevant and quick decisions. In order to enhance the reliability of data transmission and the availability of high relevant contextual information, there is a need to define efficient data summarizing and filtering mechanisms applied with a conditional scheme.

Context-aware monitoring systems require a global and full visibility of the person's context. This visibility includes recognizing daily activities and detecting abnormalities. The key challenge in such environments

\footnotetext{
* Corresponding author.

Email addresses: haider-hasan.mshali@u-bordeaux.fr (Haider Mshali), tayeb.lemlouma@irisa.fr (Tayeb Lemlouma), magoni@labri.fr (Damien Magoni)
} 
is to determine: what, when and how to monitor and analyze data related to the person's context. Our work aims at developing such an adaptive monitoring framework that can easily be integrated into health institution systems. Our system is able to evaluate the dependency level by efficiently sensing the person's behavior with optimal sampling frequencies. It can accurately detect abnormal and unusual situations whatever the person's profile. This paper is an extension of our prior work 2 in which we have shown that sensing and analyzing daily activities should be tied to the type of activity, its complexity, repeatability, and the duration required to achieve it.

The paper is organized as follows: Section 2 presents a review of related work including their limitations. The system methodology along with the framework description and proposed approaches are discussed in Section 3 while Section 4 presents our predictive model. The generation of daily scenarios is explained in Section 5. We define our context-aware, adaptive, and predictive monitoring algorithm in Section 6 . Experimental results are discussed in Section 7

\section{Related Work}

Several sensing systems were developed for monitoring and assessing the abilities of persons. However, most of them operate in isolation from the real requirements of the healthcare institutions which contributes to a high incidence of unsuccessful projects 3. [4. Mainly, we observe that none of the current approaches systematically consider the person's context (health status and behavior) as defined by the medical domain of geriatrics regarding the dependency concept. This missing link creates a high uncertainty in the adoption of such e-health systems. Therefore, it is important to improve context-aware HMS for ensuring their integration into health institutions. Taking into account models used in geriatrics can help this integration as we have shown in [5]. By considering the knowledge about the dependency profile of the person, the system can refine its services by matching them with the user's needs.

Probabilistic methods such as hidden Markov models (HMM) are used for human behavior detection by Nguyen et al. [6] and activity recognition by Trabelsi et al. [7]. However, their main drawback is the lack of hierarchy in representing the human behavior 8. Moreover, using HMMs as a time series prediction model requires a large and growing number of time sequences. In this respect, other models, such as the Grey model [9, 10, provide the prediction of future conditions with a minimum or even incomplete data. The work in [1] applies the Grey theory to the prediction of future physiological status including body temperature, blood pressure, and glucose level. Results show that the Grey Model is more efficient with more accurate forecasting values than the conventional forecasting model called Box-Jenkins model 11. Bayesian belief networks are also used in the recognition of activities in 12 and the monitoring and detection of daily activities in 13 . The key problem of Bayesian networks is that the exact probabilistic inference is intractable [8]. Suryadevara et al. propose a framework for behavior detection based on the observed data of sensors related to the daily activities [14. Time series modeling was applied to forecast the behavior and determine the wellness of elderly people. The time series analysis was based on the duration of using objects in the monitoring environment. Unfortunately, the framework considered only five simple activities. Furthermore, the result of their experimentations reveals that the forecast process is not accurate during the initial trial period of 8 weeks and the time series modeling is only possible with a minimum of 50 duration observations. A context-aware model for changes detection, using machine learning and statistical methods, is proposed in [15]. The model uses an HMM-based approach for detecting anomalies in a sequence of daily activities. A statistical process is used to identify the irregularity in routine behaviors (shift in daily routines) and a simple exponential smoothing is used to predict and detect changes in vital signs. The outputs of these processes are combined to a fuzzy model to make the final decision. The main problem with this system is the required high computational cost.

AI methods can also be used for behavior classification. The work in [16] proposes a self-organizing map to detect and recognize ADL. This system is based on a self-adaptive neural network algorithm called Growing Self-Organizing Maps (GSOM). A neural network system used for differentiating normal and abnormal behaviors based on the frequencies of ADL is discussed in [17. A predictive modeling engine proposed in [18] is mining ADL data by using machine learning algorithms with neural networks. Genetic algorithms are used for human behavior modeling and anomaly detection for the elderly in [19]. Yuan et al. propose 
a hybrid reasoning framework called Context-Aware Real-time Assistant (CARA) 20 that integrates fuzzy rule-based reasoning with case-based reasoning for designing a healthcare home automation system providing continuous health monitoring and risky situations prediction. Support Vector Machines (SVM) have been used by Kadouche et al. for activity recognition and patient behavior prediction 21. The process enables the prediction of home daily activities such as grooming, eating, sleeping, and having breakfast. Unfortunately, the results were limited to a reduced set of activities which are achieved only in the early morning.

Semantic web technologies applied to context-aware systems have also contributed to modeling and adapting behavior in smart environments and e-Health in particular. Paganelli and Giuli have proposed an ontology-based context model and context management system for health monitoring and alarm management of chronic conditions in a home-based care scenario [22]. They adopted a rule-based reasoning approach for analyzing measurement values at specific time instances to infer risks related to patient abnormal conditions. Their system, however, do not take into account system resource consumption. König et al. have studied the use of a video monitoring system for automatic event recognition for the assessment of IADL in dementia patients 23. Their system enabled automatic detection of differences in the execution time of activities between healthy subjects and mild cognitive impaired patients. However they do not integrate any other data. Stavropoulos et al. have defined DemaWare2, a framework for dementia ambient care integrating a variety of sensors, analytics and semantic interpretation 24. All data is stored in a knowledge base, enabling its semantic interpretation for further fusion, aggregation and detection of problematic behaviors. Lab trials and home care scenarios for monitoring and interventions have shown accurate data fusion and activity detection, helping clinical care. While the data collection and analysis is extensive, their system do not consider resource consumption. Also applicable to e-Health, Riboni and Bettini have created a system called COSAR for automatic activity recognition based on the integration of statistical and ontological reasoning [25. Their results have shown that this system is more accurate than purely statistical methods. It is targeted for the general population, not specifically the elderly and as such it does not consider dependency levels.

It is worth mentioning that the use of simulated data in health informatics research is common practice 26]. It has emerged as an alternative approach for developing and validating health monitoring systems 27 28]. Simulation methods can handle different activity trends thus avoiding tedious lab experiments or real-world deployments. They enable testing uncommon scenarios of everyday life (instead of waiting for unpredictable real-life apparitions), managing sensor distributions and assessing algorithms used in behavioral sciences [29]. In this context, many efforts have been made to design and use simulators imitating IADL. A study from Noury et al. uses simulated behavior for a patient living in an intelligent home 30. A fuzzy logic system is described in 31 for recognizing activities in a home environment using a set of various sensors.Their method was tested on simulated data in order to demonstrate its effectiveness. More recently, a context-aware model for monitoring ADL in ambient assisted living was presented in [15. The authors use machine learning and statistical methods for behavioral change detection and abnormality prediction. Their experimentations were applied on a dataset that was generated synthetically. Similarly in this work, our system is tested against simulated data generated by statistical methods.

\section{Framework of e-health monitoring}

In order to characterize the adaptation of our proposed monitoring system, we present the person's context within the profiling of activities and dependency evaluation. Then, we discuss our methodology in data processing. Finally, we describe, in detail, the framework and the adaptive monitoring approaches.

\subsection{Activities and person's profile}

In the context of e-health services and smart home environments, the monitoring and evaluation of the person's behavior regarding the achievement of daily activities are of high concern. This evaluation helps reveal the person's real needs and allows providing timely assistance and services which is the essence and basis of automatic monitoring. An efficient framework must ensure a smart monitoring which is reliable and flexible while being linked to the medical scales used by health professionals. This link helps the adoption 
of the proposed system by health institutions. In geriatrics, the health status and wellness of persons are measured by so-called dependency evaluation methods. For these reasons, and based on our previous work [5], we adopt the SMAF evaluation model for human activities [32. The Functional Autonomy Measurement System (SMAF) is a clinical rating scale that manually measures the functional autonomy of the elderly [32]. SMAF is used to rehabilitate individuals by providing them services and care to tackle their disabilities. In order to evaluate the person's dependency level, 29 items are used in the rating scale. Four main modalities are defined: autonomous, needs of supervision, needs for help and dependence. Five functional abilities are defined: Activities of Daily Living (ADL), Mobility, Communication, Mental Function and Instrumental Activities of Daily Living (IADL). SMAF defines 14 profiles of dependency: from $P_{1}$, which refers to autonomous persons, to $P_{14}$, which refers to completely dependent persons 32 .

\subsection{Data processing issues}

We propose the use of a non-uniform interval that we call a validity period. The validity period is an amount of time evaluated for each activity and determined by the condition of the person's profile (dependency level) and historical record. This leads to define different classes of validity periods for the monitored activities. For each activity, the variability of the period depends on the health status and person's record (normal behavior, chronic disease, etc.). The validity period is influenced by the detection of abnormal behavior of the elderly in independent living. Such changes in patterns allow the HSH to alert caregivers who will provide personalized assistance and needed services. Periods are context-aware, which means they are estimated on the basis of the patient's profile. More specifically, we consider the dependency level and the person's history to set the validity of data and determine the frequency for requesting data.

In order to address the issues discussed previously and fulfill the identified requirements, we propose an adaptive context-aware monitoring framework with a conditional processing scheme. The framework provides an efficient monitoring thanks to the determination of an optimal and context-aware sensing, a dynamic update of the sensing frequencies and the use of prediction regarding the person's behavior. Hence, the system will be able to detect any change and to drive therefore the used sensors to an optimal monitoring. Fig. 1 presents our request-driven monitoring scheme. The elderly profile, which includes the dependency level and historical record, represents a key factor to adapt the monitoring periods and to optimize the sensor nodes for reducing sensing and processing only highly relevant data. Consequently, our proposed system solves the main issues associated with traditional monitoring schemes and improves the dependency evaluation models used in geriatrics. Furthermore, our approach ensures an efficient processing of the collected data and analysis for the prediction of the person's behavior.

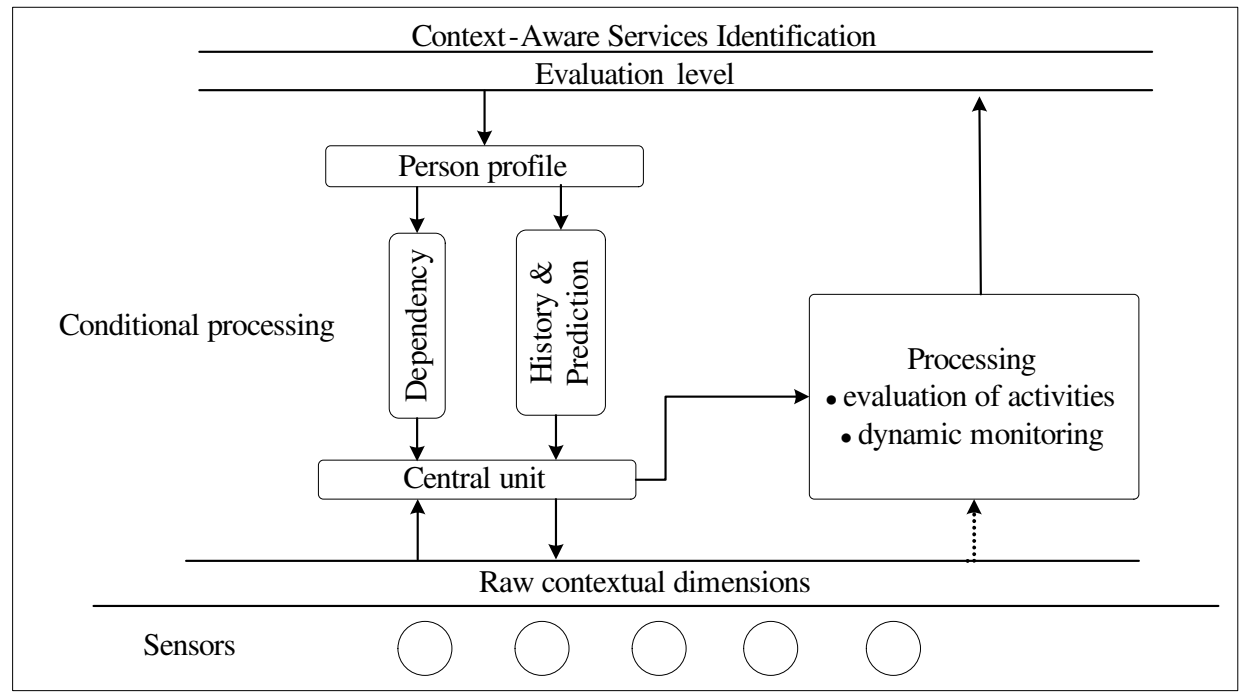

Figure 1: Request-driven monitoring scheme. 


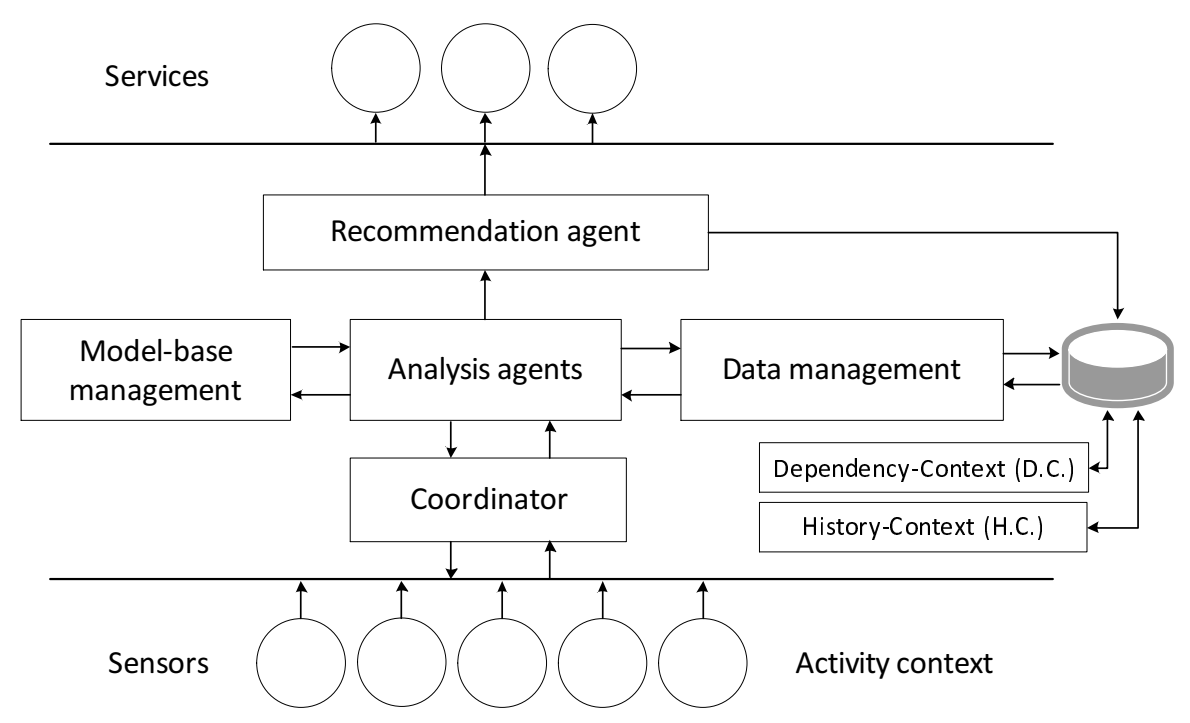

Figure 2: Components of the framework for the e-health monitoring system.

\subsection{Framework description}

Daily activities represent the context in our context-aware approach. It is used as the base layer for the different functionalities such as sensing, processing, and recommending services (Fig. 2). The person evolves in a smart space with adequate sensors. Data is collected and transmitted by sensors in a continuous or periodic way. The coordinator analyses and processes the received data. Streams coming from different sources are handled by the data management system, which is able to apply database primitives such as queries and updates. The analysis agents consider the person's profile which includes dependency-context (D.C.) and history-context (H.C.) for a specific period of time. The first inference is applied to set up the monitoring mode. This is achieved thanks to the connection between the analysis agents and the model-base management. The latter selects the geriatric model which is in turn considered in the data management and combined with input data to adjust the monitoring. Finally, sensing and processing data will result in recommending e-health services adapted to the person's situation.

\subsection{Used approaches for an adaptive monitoring}

In order to model the complex human behavior using realistic and adaptable models, our adaptive context-aware monitoring system defines and combines two approaches: the evaluation of activities approach and the dynamic monitoring approach.

\subsubsection{Evaluation of activities}

The aim of this approach is to evaluate each monitored activity separately. This approach is particularly important during the initial monitoring phase and helps to learn the person's behavior evolution in performing the daily activities. We consider a rich set of activities linked to the scales used in geriatrics. This set includes the SMAF activities and extends them with additional ones, for example, eating, toileting, reading and watching TV. We associate, during the initialization of the system, a set of metadata to each activity depending to its complexity and the necessary frequency for monitoring it. Such metadata include the category of each activity, the monitoring frequency, duration of monitoring, possible used sensors, and scores used to determine the optimal monitoring mode to be applied. Table 1 presents a subset of the considered activities and their categories (first and second columns) with the linked information. The frequency is used to specify when the monitoring of our system should start with a varying range of sensing frequency ( $x$ values) while the duration specifies how long the monitoring must be performed. The classes of activities (fifth column of the table) are defined to distinguish the level of consumption of the resources (e.g., network 
Table 1: Evaluation of activities: categories, sampling frequencies and methods, classes of sensors, and evaluation scores.

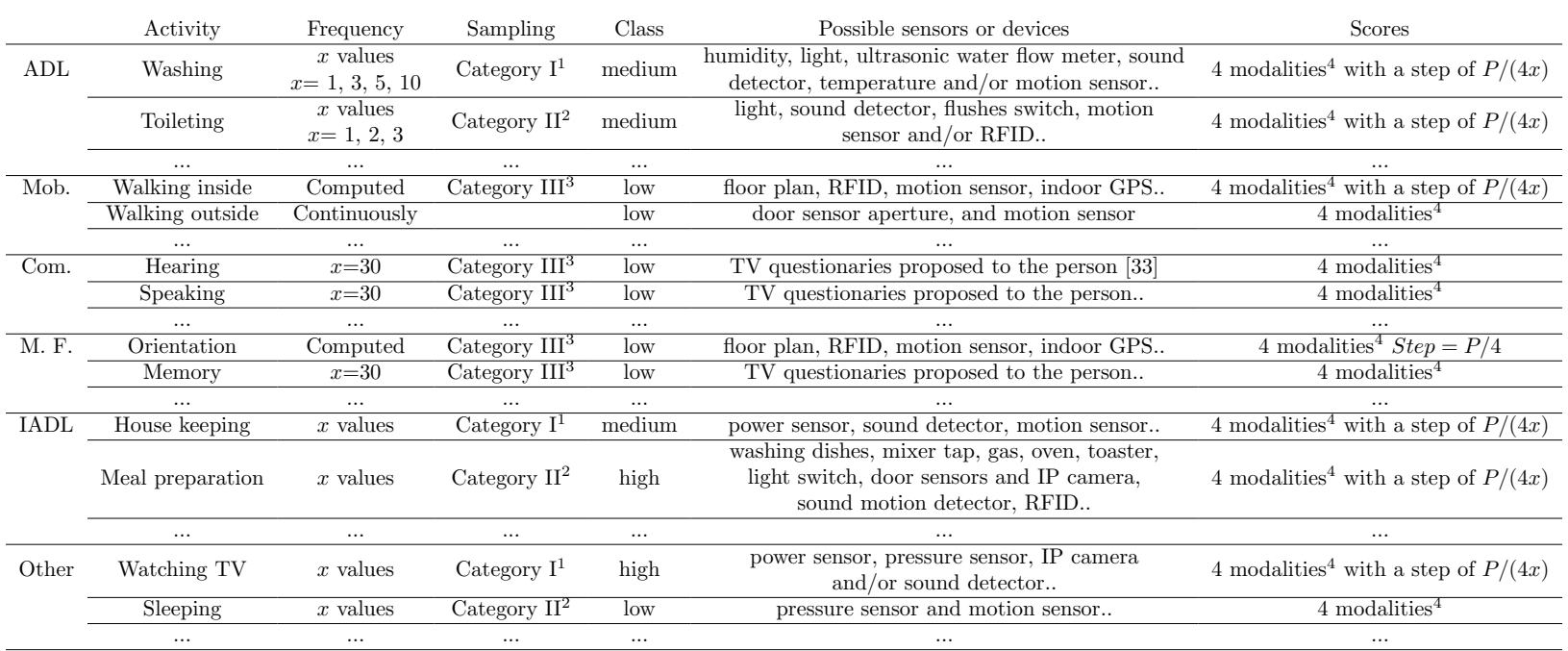

traffic and bandwidth, power, etc.). The used classes refer to those defined in Section 7.2. The sixth column of Table 1 shows some possible sensors and devices that can be used to monitor each corresponding activity. As used in geriatrics, scores are used to quantify the ability of the person's to achieve the activity. To facilitate the evaluation of activities using Table 1, we explain in the following, how the sampling methods (column 4) are applied and how the scores (last column) are evaluated in the adaptive system.

In order to start the monitoring mode, we first set the monitoring values for each activity regarding the frequency, using the $x$ value (column 3), and the duration. The activities are classified into three main categories of monitoring (column 4). For instance, in Category II which includes activities such as toileting, the initial monitoring frequency (i.e., the $x$ value) can be one of the different values $(1,2$ and 3$)$ and the monitoring duration is 24 hours. For example, for a given activity, if $x$ is fixed to 2, this means that we will trigger a new monitoring every 2 days. Each new monitoring takes 24 hours as mentioned according to Table 1 (see Category II). For Category I, including activities like washing (line 1 of Tab. 1), if the selected frequency ( $x$ value) is 3 and the duration is always active till the activity occurs, so the next round of monitoring will be triggered after the 3 days.

In Category II, in order to evaluate the activities such as toileting, eating, and sleeping, the activity is monitored, as mentioned, each 2 days during 24 hours. This leads to a total of 15 results during $P=30$ days. Each single result indicates if the person succeeds or fails to perform the activity. Results are progressively used to judge the person's ability through the duration of the monitoring. In Category I (e.g. washing, house keeping, and dressing), it is only required to check if the activity is achieved or not in order to be considered. For Category III, we use the relationships between activities. For instance, memory is associated with the ability to remember to take medication. The behavior is checked as follows. If the number of performed activities is less or greater than the expected values (mainly regarding the repeatability and durations), an abnormality is detected. Thus, the monitoring is extended with an extra time. Otherwise, the system considers single results that quantify the behavior, if the number of achieved activities is more than or equals values defined in geriatrics. For example, regarding the toileting, if no abnormality is detected and if the number of toileting is greater than or equals to 2, a single result will be kept for a given period, such as for one day.

Based on real-world geriatric models, the person's ability to perform activities (i.e., the degree of dependency) is evaluated using scores. Our adaptive system regularly evaluates the degree of dependency each period of time (called $P$ ). During this period $P$ and for each activity, the system counts how often the person correctly achieves the activity. The counter, used in our algorithm, is called activityResults. The counter is updated depending on the frequency of sensing. Specifically, it is updated each $x$ period. This 
Table 2: Dynamic monitoring modes.

\begin{tabular}{|c|c|c|c|c|c|}
\hline & ADL & Mobility & Communication & Mental Functions & IADL \\
\hline Profile $_{1}$ & $x=x_{0} / 1$ (initial $\left.x\right)$ & $x=x_{0} / 1$ & $x=x_{0} / 1$ & $x=x_{0} / 1$ & $x=x_{0} / 1$ \\
\hline Profile $_{2}$ & $x=x_{0} / 1$ & $x=x_{0} / 1$ & $x=x_{0} / 1$ & $x=x_{0} / 1$ & $x=x_{0} / 1$ \\
\hline Profile $_{3}$ & $x=x_{0} / 1$ & $x=x_{0} / 1$ & $x=x_{0} / 1$ & $x=x_{0} / 1$ & $x=x_{0} / 2$ \\
\hline Profile $_{4}$ & $x=x_{0} / 1$ & $x=x_{0} / 1$ & $x=x_{0} / 1$ & $x=x_{0} / 1$ & $x=x_{0} / 2$ \\
\hline Profile $_{5}$ & $x=x_{0} / 1$ & $x=x_{0} / 1$ & $x=x_{0} / 1$ & $x=x_{0} / 1$ & $x=x_{0} / 3$ \\
\hline $\begin{array}{l}\text { Profile }_{6} \\
\end{array}$ & $x=x_{0} / 1$ & $x=x_{0} / 1$ & $x=x_{0} / 1$ & $x=x_{0} / 1$ & $x=x_{0} / 3$ \\
\hline Profile $_{7}$ & $x=x_{0} / 1$ & $x=x_{0} / 1$ & $x=x_{0} / 1$ & $x=x_{0} / 2$ & $x=x_{0} /(3-1)$ \\
\hline Profile $_{8}$ & $x=x_{0} / 1$ & $x=x_{0} / 1$ & $x=x_{0} / 1$ & $x=x_{0} / 2$ & $x=x_{0} /(3-1)$ \\
\hline Profile $_{9}$ & $x=x_{0} / 2$ & $x=x_{0} / 2$ & $x=x_{0} / 1$ & $x=x_{0} / 1$ & $x=x_{0} /(3-2)$ \\
\hline Profile $_{10}$ & $x=x_{0} / 2$ & $x=x_{0} / 1$ & $x=x_{0} / 1$ & $x=x_{0} / 2$ & $x=x_{0} /(3-2)$ \\
\hline Profile $_{11}$ & $x=x_{0} / 2$ & $x=x_{0} / 2$ & $x=x_{0} / 1$ & $x=x_{0} / 2$ & $x=\inf$. \\
\hline Profile $_{12}$ & $x=x_{0} / 3$ & $x=x_{0} / 2$ & $x=x_{0} / 1$ & $x=x_{0} / 2$ & $x=\inf$. \\
\hline Profile $_{13}$ & $x=x_{0} / 3$ & $x=x_{0} / 3$ & $x=x_{0} / 1$ & $x=x_{0} / 2$ & $x=\inf$. \\
\hline Profile $_{14}$ & $x=x_{0} / 3$ & $x=x_{0} / 3$ & $x=x_{0} / 2$ & $x=x_{0} / 3$ & $x=\inf$. \\
\hline
\end{tabular}

means, after each $x$ period, the system senses the person's environment to checks if the person succeeds to perform the activity. If it is the case, the system increments the activityResults counter. The value of the counter is reset at the end of the $P$ period. The following example illustrates this method. Consider an activity $A$ required to be achieved once a day by the monitored person. If $P=30$ days, $x=3$ days and activityResults $=9$, this means the following. The system calculates the dependency level regarding the activity $A$ each 30 days; thanks to sensors, it counts how often $A$ was achieved correctly each 3 days. The example shows that the activity $A$ was achieved correctly by the monitored person 9 times (activityResults $=9)$ during the last 30 days.

The dependency scores can be one of the following values: 0 (Autonomous or A), -1 (Supervision or $S),-2($ Need help or $H)$, and -3 (Dependency or $D)$, see section 3.1. To connect the activity counter (activityResults), explained previously, to these four modalities (i.e., $A, S, H$ or $D$ ), we define four linear intervals. Intervals are defined with a step of $P / 4 x$ and associated to the four modalities as follows: $D \equiv$ $[0, P / 4 x[; H \equiv[P / 4 x, 2 P / 4 x[; S \equiv[2 P / 4 x, 3 P / 4 x[$ and $A \equiv[3 P / 4 x, P / x]$. For instance, if we consider the previous example (i.e., with $P=30$ days, $x=3$ days and activityResults $=9$ ), then the following intervals are considered: $D \equiv[0,2.5[; H \equiv[2.5,5[; S \equiv[5,7.5[$ and $A \equiv[7.5,10]$. Note that the value of activityScore (i.e., 9) belongs to the $A$ interval. Hence, the person is evaluated autonomous in achieving the activity $A$ for the last 30 days.

\subsubsection{Dynamic monitoring}

The main objective of our dynamic monitoring approach is to determine the optimal degree of data sensing (i.e., frequency) in order to avoid unnecessary data, which is usually present in traditional monitoring systems. Moreover, we aim to avoid the exaggerations of the existing dependency evaluations that consider all the activities at all the dependency levels even in severe ones [5. Request-driven monitoring approaches, as in [34, represent a good candidate to optimize the blind continuous monitoring. Therefore, for the sake of optimizing the monitoring mode, our adaptive monitoring considers the dependency evaluation of the person as the basis for increasing or decreasing the monitoring frequency. Our approach uses the initial frequency ( $x$ value) discussed previously. It performs an overall evaluation of the person abilities based on geriatric models. The evaluation is achieved using the SMAF model (scale) which applies a summation of all activity scores and identifies the global evaluation (i.e., iso-SMAF profile) [32].

The idea is to provide a dynamic monitoring frequency that dynamically adapts the initial $x$ value depending on the person's dependency level (from autonomy to severe dependency) and relying on the nature of the monitored activities such as ADL and IADL (see Table 2). To reach this objective, and based on our work in [5], we adopt the SMAF model used in real-world evaluations [32] (first column of Table 2). As presented previously, SMAF defines 14 dependency levels (profiles) from profile 1, which refers to autonomous persons, to profile 14, which refers to completely dependent persons. These levels are based on the evaluation of the activities using the five functional abilities: activity of daily living (ADL), mobility, communication, mental function and instrumental activity of daily living (IADL).

Table 2 shows the dynamic updates of the $x$ sampling frequency regarding the person's profile, which is periodically evaluated. The selected frequency, $x$, is related to the type of monitored activity and depends 
on the current person's profile. Thus, the monitoring can be increased, decreased or even stopped. Based on the SMAF model, each profile implies a dependency level which may vary from autonomy to dependence. The adaptive system uses a default value of $x$ (called $x_{0}$ ) when it monitors autonomous persons. When a decline of the health conditions occurs, the $x$ value decreases. For example, for persons who belong to profile 1 (row 2 of Table 2), the monitoring uses an initial set of values $x_{0}$ associated to each activity. For instance, for the washing activity (i.e., ADL category, column 1 of Table 2), the sampling frequency is set to $x_{0}$ where the value is 3 for the washing activity (see Section 3.4.1). Overall, the system requires more sensing for higher dependency profiles. This rule is true except for profiles $P_{7}$ to $P_{14}$ regarding IADL. The reason for this choice is related to the nature of the IADL activities, which are lost first by persons. When a person becomes unable to perform the IADL activities, assistance is required in order to perform the other type of activities. Therefore, starting from profile $P_{7}$, the adaptive system increases the monitoring for the IADL activities. As mentioned before, the monitoring can be stopped (i.e., $x=$ infinity). This concerns only IADL and occurs if, and only if, the person starts to be completely dependent for the majority of activities. More specifically, from profile $P_{11}$ to $P_{14}$ in the SMAF model. This situation is called long-term care facilities (LTCF).

\section{Prediction of person's behavior}

Changes in activities performance can be determined using durations, frequencies, and absence of activities. Despite the unstable intraday behavior of persons and the uncertainty of the environment, data series including the person's history help to forecast future behaviors. To that end, we use the Grey theory [9, 10]. We aim to learn the behavioral patterns with the minimum and most related data. The model ensures a prediction of the health condition based on the person's behavior and using the knowledge related to the energy consumption required for monitoring each activity to detect abnormal situations. Consequently, the system provides a proactive attention and can trigger notifications to caregivers if there is a risk of decline.

\subsection{Forecasting model}

The Grey Model (GM) is widely used in the prediction of systems coming with incomplete data and uncertain concepts. GM represents an efficient tool with only short learning (training) periods. GM uses $n$-order differential equations and a set of variables of cardinal $h(\mathrm{GM}(\mathrm{n}, \mathrm{h}))$. We use the single variable first order model GM $(1,1)$ to learn and predict the behavior and level of dependency trends. GM $(1,1)$ is summarized as follows [9. The system considers an initial non-negative time sequence of data:

$X^{(0)}=\left\{x^{(0)}(1), x^{(0)}(2), . ., x^{(0)}(n)\right\}$

Based on the initial sequence $X^{(0)}$, a new sequence $X^{(1)}$, called accumulated sequence, is generated by applying the accumulated generating operation (AGO) in order to smooth the randomness.

$X^{(1)}=\left\{x^{(1)}(1), x^{(1)}(2), . ., x^{(1)}(n)\right\}$

Where $\quad x^{(1)}(k)=\sum_{i=1}^{k} x^{(0)}(i), \quad 1 \leq k \leq n$

The generated mean sequence $Z^{(1)}$ is derived from $X^{(1)}$ using the mean value of each two consecutive terms.

$Z^{(1)}=\left\{z^{(1)}(2), z^{(1)}(3), . ., z^{(1)}(n)\right\}$

Where $\quad z^{(1)}(k)=\frac{1}{2} x^{(1)}(k)+\frac{1}{2} x^{(1)}(k-1), \quad 2 \leq k \leq n$ 
The first order differential equation of $\operatorname{GM}(1,1)$ is defined in the following,

$x^{(0)}(k)+a z^{(1)}(k)=b, \quad 2 \leq k \leq n$

Thus, the whitening equation is $\frac{d x^{(1)}}{d t}+a x^{(1)}=b$

Let $Y$ and $B$ be: $Y=\left[\begin{array}{c}x^{(0)}(2) \\ x^{(0)}(3) \\ \vdots \\ x^{(0)}(n)\end{array}\right], B=\left[\begin{array}{cc}-z^{(1)}(2) & 1 \\ -z^{(1)}(3) & 1 \\ \vdots & \vdots \\ -z^{(1)}(n) & 1\end{array}\right]$

Then, $a$ and $b$ parameters can be found as follows: $\quad[a, b]^{T}=\left(B^{T} B\right)^{-1} B^{T} Y$

According to the whitened equation of $\operatorname{GM}(1,1)$, the solution of $X^{(1)}$ at time $k$ is:

$x_{p}^{(1)}(k+1)=\left[x^{(0)}(1)-\frac{b}{a}\right] e^{-a k}+\frac{b}{a}$

Consequently, to obtain the predicted value of the initial data row at time $(k+1)$ we use

$x_{p}^{(0)}(k+1)=\left[x^{(0)}(1)-\frac{b}{a}\right] e^{-a k}\left(1-e^{a}\right)$

For all the considered activities, we calculate the predictive values (duration and frequency) by applying the previous model on the initial data sequence, which is represented by the input values. Algorithm 1 presents an example of prediction about the required duration of activities. Initial data include the sequence of durations that were used, by the person, to achieve the daily activities. If the data raw size is very small (less than 3), the system considers the previously observed durations. Otherwise, the Grey model is applied to predict next values. Fig. 3 shows an example of the observed (input) values and the predicted trend of monitored activities. The example considers the toileting activity in terms of durations and frequencies (i.e., the number of performed activities).

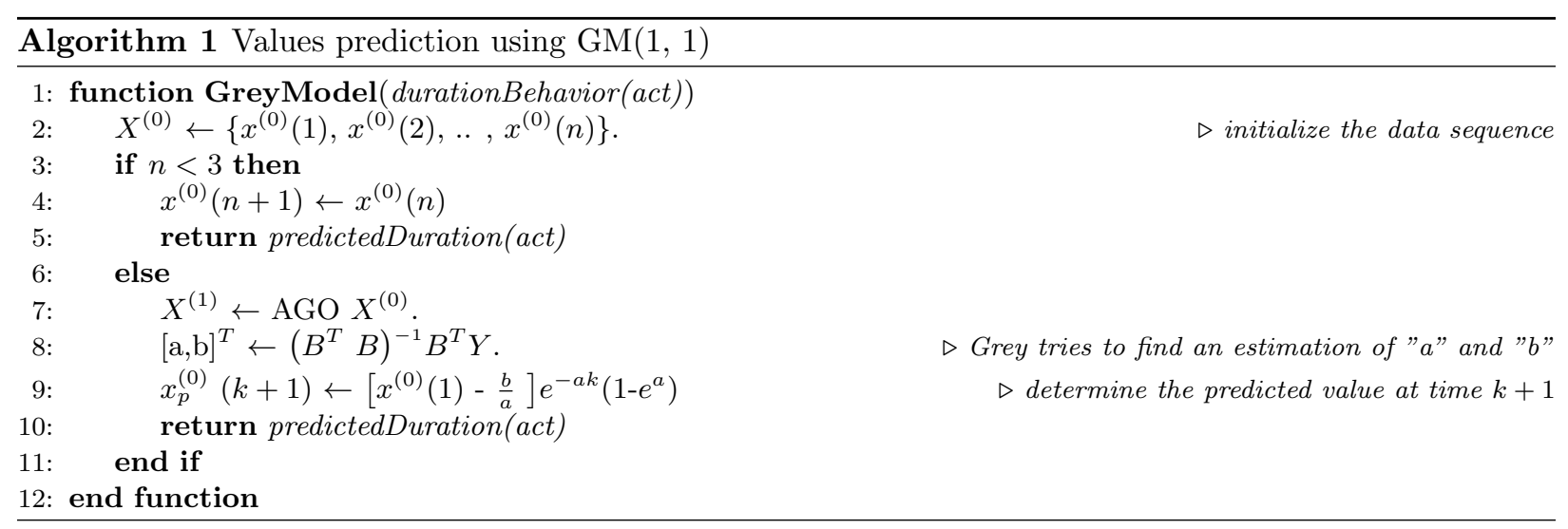



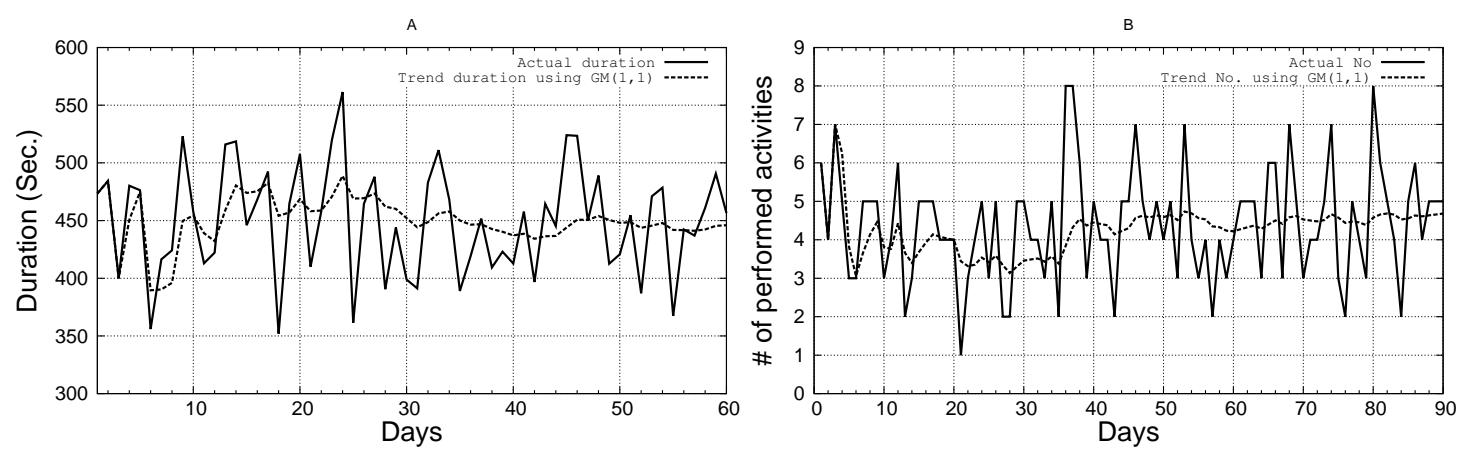

Figure 3: Comparison between the input (observed) values and the predicted values using GM(1,1) for the toileting activity.

\subsection{Behavior change detection}

Using a minimum amount of sensed data and short training periods, our proposed monitoring system categorizes the behavior of the elderly as normal (regular) or abnormal (irregular). After the determination of the standard deviation $\sigma$ of the input values and the Grey values using Eq. 11. we get a range of forecast between a lower (minimum) and upper (maximum) limits where the Min range is Grey value - $\sigma$ and the Max range is Grey value $+\sigma$. The duration and frequency for each activity are checked with maximum/minimum ranges of forecast. A given situation is considered as a normal behavior if the input values for the activity (i.e., duration and frequency) fit with the forecasting range [Grey value $-\sigma$, Grey value $+\sigma]$. Otherwise, the observation is categorized as an anomalous situation. It is worth noting that the combination of the standard deviation (SD) with GM $(1,1)$ values represents a good indicator for the detection of the behavior changes.

Unfortunately, this indicator is not sufficient to judge if a behavior change represents a real abnormality or not. By relying only on SD, some normal situations could be seen as abnormal if they are compared to the majority of inputs. Similarly, real abnormalities can be ignored if they occur in a series of abnormal values. Unlike expert data, SD values depend only on relative comparisons between observed values whatever the performed activity. Expert data are based on medical knowledge and experiments that identify real risks, which depends widely on the nature of the activity. For instance, with some profiles and illnesses, mean values and acceptable deviations for some activities should be properly configured in order to avoid false detections of abnormalities. Consequently, input expert data (e.g., coming from health professionals) are needed during the setup of the system in order to moderate the SD impact. These inputs enrich the system with the ability to determine if a detected change represents really an abnormality that could be risky for the subject.

\section{Generation of daily activities scenarios}

In order to evaluate the efficiency of our adaptive system in learning and predicting the person's behavior with efficient sensing frequencies, we need to experiment a series of rich and realistic scenarios that describe the daily living activities performed by a single person with different levels of dependency. The generation of simulated ADL scenarios can provide sufficient data to help the design and test of approaches defined for e-health and assisted living systems [27. One key requirement for such simulation is that the daily life scenario should consider events and human activities in a realistic way. This means to be as close as possible to the real life of an elderly or a dependent person. Moreover, scenarios for long periods allow the evaluation of various aspects of our proposal such as the dynamic monitoring and the health decline impact. Real-life platforms require complex implementations including an important number of sensors required to consider a long list of activities (e.g. those defined in geriatric models). Hence, in order to make a proposed system ready for the operational environment, a real-life implementation requires a long-time testing that implies the life of subjects. This represents a lack of flexibility regarding the aim to vary the used sensors and may 
Table 3: Comparison between real-world datasets and our monitoring dataset.

\begin{tabular}{|c|c|c|c|c|c|c|}
\hline Dataset & Activities $[32 \|$ & Duration & Geriatric models & Sensor heterogeneity & Time slices for activities & Profile changes \\
\hline $\begin{array}{c}\text { Kasteren } \\
(\mathrm{A}, \mathrm{B} \text { and } \mathrm{C}) \\
\text { 35] }\end{array}$ & ADL (3 activities) & $\begin{array}{c}22 \text { days }(\mathrm{A}), 12 \text { days }(\mathrm{B}) \\
17 \text { days }(\mathrm{C})\end{array}$ & no & low class & $\begin{array}{c}33,120(\mathrm{~A}), 17,280(\mathrm{~B}) \\
24,480(\mathrm{C})\end{array}$ & no \\
\hline $\begin{array}{c}\text { Ordonez } \\
\text { (A and B) 36] }\end{array}$ & ADL (3 activities) & $\begin{array}{l}14 \text { days }(\mathrm{A}) \\
21 \text { days }(\mathrm{B})\end{array}$ & no & low class & $\begin{array}{l}20,160(\mathrm{~A}) \\
30,240(\mathrm{~B})\end{array}$ & no \\
\hline MIT 37] & $\begin{array}{l}\text { ADL ( } 4 \text { activities }) \\
\text { IADL ( } 2 \text { activities })\end{array}$ & 15 days & no & $\begin{array}{l}\text { low, medium, } \\
\text { and high classes }\end{array}$ & $\begin{array}{c}690 \\
\text { (for annotated activities) }\end{array}$ & no \\
\hline $\begin{array}{c}\text { CASAS Kyoto } \\
\text { Daily life } 2010 \text { 38 }\end{array}$ & $\begin{array}{l}\text { ADL ( } 4 \text { activities) } \\
\text { IADL ( } 2 \text { activities) }\end{array}$ & 8 months & no & low class & 3,741 & no \\
\hline Our dataset & $\begin{array}{l}\text { ADL (5 activities) } \\
\text { IADL (5 activities) }\end{array}$ & 5 years & $\begin{array}{c}\text { yes } \\
(\mathrm{SMAF})\end{array}$ & $\begin{array}{c}\text { low, } \\
\text { medium, } \\
\text { and high classes }\end{array}$ & $\begin{array}{c}13,933 \text { ( } 1 \text { year }), 14,240 \text { (1 year) } \\
10,981 \text { ( } 1 \text { year }), 7,0238 \text { (1 year }) \\
11,436(1 \text { year })\end{array}$ & $\begin{array}{c}\text { yes } \\
\text { (4 changes) }\end{array}$ \\
\hline
\end{tabular}

represents a risk for the person if the algorithms should evolve during the testing. Another requirement for the input scenarios is to consider abnormal situations that may occur during the monitoring of the person. To fulfill the identified requirements and gain an efficient evaluation, it's necessary to select realistic, rich and flexible scenarios that take into consideration various human activities monitored for long periods.

Simulating realistic daily activities is a challenging task since the achievement of activities is usually subject to a number of factors such as basic needs, lifestyle, physical, and mental abilities [27. The scenarios should be impacted by the level of dependency and the different persons' profiles. Unfortunately, real-life monitoring (within a reasonable time, e.g., one year) cannot provide a good testing environment (Table 3). Such monitoring will not allow the evaluation of our proposal regarding our focus on adaptability and predictability. Moreover, abnormalities and changes in the dependency level should occur during the monitoring in order to validate the ability to detect them. Abnormalities are risky for real subjects and significant changes of the dependency degree, as observed in geriatrics, takes a very long time. To the best of our knowledge, existing real-world datasets monitor persons belonging to the same profile with the same dependency level. Table 3 presents the most complete existing datasets regarding the number and categories of monitored daily activities, their time slices (duration), and the consideration of several sensor classes. For the resource consumption evaluation, only a limited set of sensors (or sensors belonging to the same class) was used in the explored datasets. This is why we require considering heterogeneous classes of sensors in terms of energy consumption and network use. All the investigated real-world datasets that monitor elderly did not consider a rich set of activities (including ADL, IADL and additional activities) for long periods. They involve a subset of activities (or simple human actions) and are not linked to geriatric models, used by health professionals. Hence, they fail in providing a good visibility regarding the person's context.

There are several simulation studies describing daily behavior profiles, which often provide limited information such as regarding the spatial context (i.e., room transitions). The authors in [39] and [30] used Markov Models to estimate the room of the person. The work used the same model in different periods for each day. Consequently, the scenario of every day has similar possible results [40. The simulation model defined in [30. ignores the sequence of activities, which can describe a lifestyle of a person. Indeed, the used probability of performing an activity at time $t+1$ depends only on the activity achieved at time $t$.

In our work, we are based on our strategy of scenarios generation discussed in [41] based on Markovian models. We aim to provide a long and rich realistic sequence of activities that can be achieved by elderly with different levels of disabilities including autonomous persons. To achieve this objective, we were based on the variable-length Markov class (VMM [42]) which helps in increasing the expressivity level while generating the sequences of daily living activities. Such a sequence is denoted as follows: $s=a_{1}, a_{2}, \ldots, a_{l}$ where $l$ is an order greater than one. Activities, $a_{i}$, are the human actions performed for the different activities as defined in geriatrics. Thanks to the results of our previous study in [5], we were mainly focused on the SMAF model. We associate, for each action, a startingTime and a variable (pseudo-random) duration activityDuration in the range $\left[a D_{\min }, a D_{\max }\right]$. A pseudo-random transition time transitionTime $\left(a_{i}, a_{j}\right) \in$ $\left[t T_{\min }, t T_{\max }\right]$ is used from the end of each action $a_{i}$ to the starting time of an action $a_{j}$. To keep outputting realistic sequences, we define 5 transition matrices which correspond to the following day periods: from 8.00 am to $11.00 \mathrm{am}$, from $11.00 \mathrm{am}$ to $2.00 \mathrm{pm}$, from $2.00 \mathrm{pm}$ to $5.00 \mathrm{pm}$, from $5.00 \mathrm{pm}$ to $10.00 \mathrm{pm}$, and from $10.00 \mathrm{pm}$ to $8.00 \mathrm{am}$. In addition, we define two other matrices for particular periods and days such as for Friday and Sunday since these days could include some specific activities. Most activities that can 
be achieved by the person during a given period are associated with the highest probability. For instance, taking a shower in the morning, having dinner in the evening, possible house keeping in Friday and leaving the home for a long period in Sunday. The defined matrices and generated scenarios can be accessed online in [43. We propose the use of the Markov property in our generation strategy of scenarios. We define

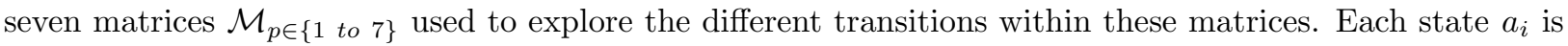
a possible human action or activity as defined in the real-world geriatric models. Next actions, $a_{j}$, follow the probability $P\left(a_{j} \mid a_{i}\right)=\mathcal{M}_{p}\left(a_{i}, a_{j}\right)$. The definition of probabilities $P$ is performed for each person's profile as defined in the SMAF model. When a new action $a_{j}$ is selected, it is appended to the sequence $s$ which is the sequence of the current activities performed by the person.

Even if the used probabilities are strongly related to the real-world SMAF model, relying exclusively on the probabilities of transitions provides what is knows as a random walk approach. In order to improve the generation, it is important to avoid the possible drawbacks of the random walk. We cite, mainly, the possible consideration of less probable sequences and the lack of control during the construction of sequences. Indeed, an absolute random walk could end up with a sequence that ignores a required activity in a given day period (e.g. morning or afternoon). This may occur even if the person has the ability to achieve the activity. Another example is the possible generation of a sequence that takes a long time that significantly exceeds a given day period. To tackle these issues, we control the previous generation process by adopting a set of constraints. This approach leads to define a pseudo Markovian model, where sequences $s$ rely on the transition probabilities under certain conditions. Each transition, from a current state to a possible state, is checked using the introduced constraints. If the transition to a new state violates, at least, one constraint, the random walk is reoriented to another possible state.

Constraints and matrices $\mathcal{M}_{p}$ guarantee, by construction, the following properties: (a) a finite generation and convergent sequences, and (b) transitions that are faithful to the person's dependency level as defined in SMAF profiles [32]. We define two main constraints: the frequency of occurrence $f\left(a_{i}\right) \in\left[f_{\min }, f_{\max }\right]$ of some particular activities and the duration $s d$ of a sequence. Thus, a given activity can occur at least $f_{\min }$ (that could be null) and at most $f_{\max }$. This constraint controls how much particular activities should appear in a sequence. For example, the number of grooming and eating for an autonomous person. When all the non-null frequencies are satisfied, the process stops when the duration of the whole generated sequence reaches or starts to exceed the $s d$ value. The value of $s d$ is computed as follows:

$s d=\sum_{i=1}^{l}$ activityDuration $\left(a_{i}\right)+\sum_{i=1}^{l-1}$ transitionTime $\left(a_{i}, a_{i+1}\right)$, with a sequence $s=a_{1}, a_{2}, \ldots, a_{l}$.

\section{Monitoring algorithm}

In Fig. 1 and Fig. 2, we summarize our methodology and context-aware approach to guarantee a monitoring system for elderly and dependent persons regarding their ADL. The main idea is to benefit from the previously discussed approaches, in order to identify an efficient monitoring mode and make it dynamically adapted to the current person's situation. The proposed algorithm simulates data series with a time evolution (variable $i$ ) and uses the discussed approaches on various one-year scenarios (Algorithm 3. lines 2-3). All the considered activities, categorized in Section 3.4.1, are associated with a monitoring instant (MTime) which depends on the sensing frequency $x$. The $x$ value varies according to the nature of the activity and the monitoring mode. The sensing is regularly updated and depends on the evaluation of person's context (profile). To evaluate the person's state, we use the scores associated with different activities (see Algorithm 3. lines 11, 23 and 39). It is worth repeating that, scores are tested with the four modalities: $A, S, H$ and $D$ as we can see in the SMAFScore function (line 53), presented in Algorithm 2 and described in Section 3.4.1. The person's profile is then computed using the computeSMAFProfile function (line 55). The person's profile determines the new $x$ value and monitoring instant (MTime) for each activity (line 56) using the DynamicMonitoring procedure (Algorithm 2). Without long training period and even with data sequences that are incomplete, our algorithm starts to approximate the behavior of the daily life and predict next values. For this purpose, we mainly use the duration and repeatability in achieving the different activities (using Duration() and actNumber() functions in Algorithm 3 . lines 10 and 35 for instance). When the observed and predicted behavior are similar, the regular and continue monitoring mode remains unchanged (lines 12 and 40). Otherwise, the system detects abnormalities (lines 15, 25 and 
37) and applies a new mode of continuous monitoring (lines 14, 33 and 36). The new mode is stopped once the behavior becomes as usual. As we can notice, the proposed Algorithm relies on predictive functions that use many factors such as durations, repeatability and power consumption required by the activities. As presented before, Algorithm 1 gives an example of predicting values regarding the duration required in performing the person's activities.

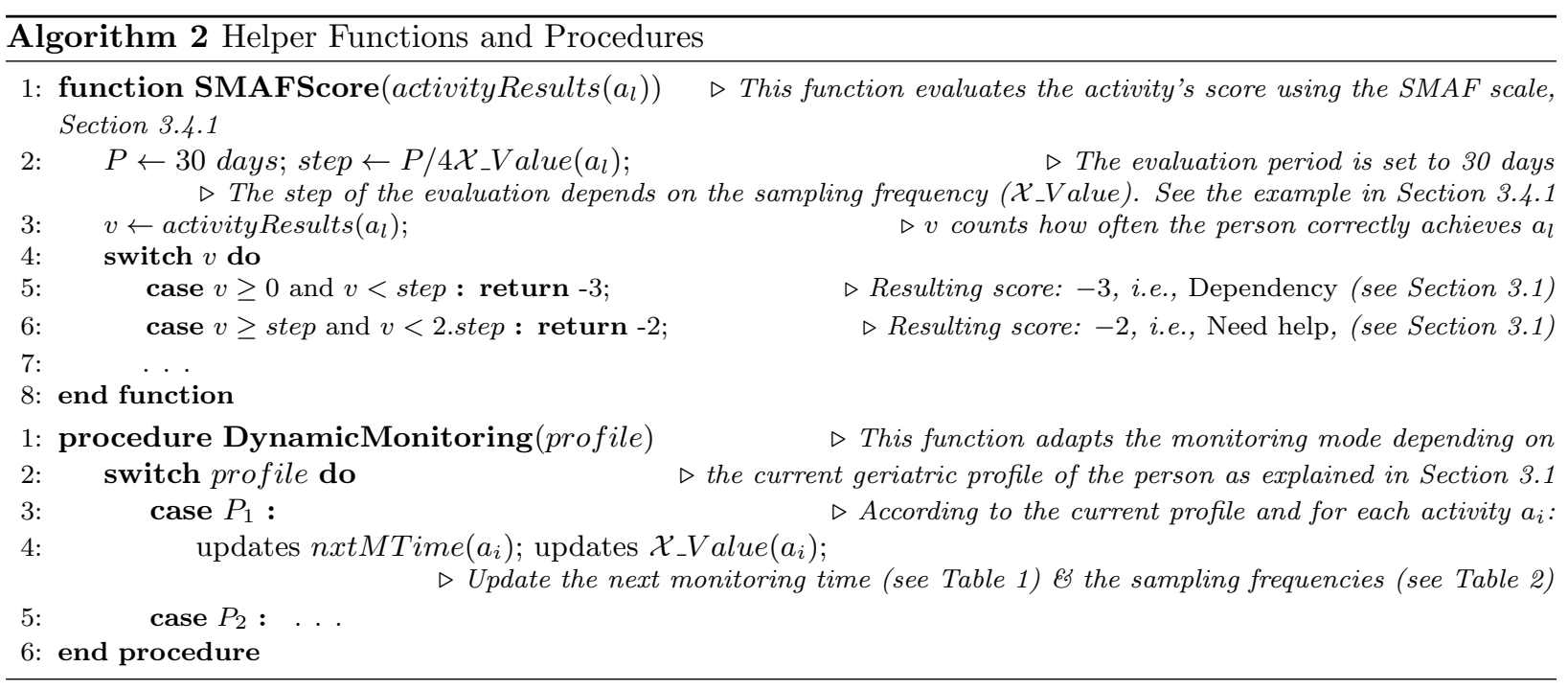

\section{Experimental results}

In accordance with the proposed approaches and developed framework for an efficient context-aware ehealth monitoring system discussed previously, we conducted various experiments to evaluate the proposed system in the terms of (i) modeling of the daily life behavior and the generation of dataset series; (ii) testing the monitoring algorithm and its adaptability; (iii) efficiency of sensing frequencies selection; and (iv) performance and accuracy evaluation of the prediction regarding the evolution of the person's conditions.

\subsection{Datasets description}

The used dataset is generated based on the pseudo variable-length Markovian model that we have defined (Section 5) and developed using the Matlab environment. This simulated dataset describes the performances of the elderly regarding the achievements of the daily life activities. Scenarios included in the dataset involve sequences of activities achieved, during a whole year, by an elderly with different levels of dependency. The simulation produced several scenarios within the same person's profile (profile $P_{1}$ ) and with profile changes representing the person's loss of abilities. The representation of the experimental dataset involves different formats, codes, and names for actions and for high-level activities composed of atomic actions [43. An overview can simply be presented using time series as [Day's number, Starting time, Day's number, Ending time, Activity name]. Table 4 shows an example.

In order to satisfy the activities (items) listed in the SMAF model using the five aspects of functional abilities, our dataset simulation includes most of the basic activities and related events such as eating in ADL, meal preparation in IADL and going outside for mobility. Other items, such as hearing, speaking, and memory are considered in communication and mental functions, do not appear in the dataset but are deduced based on the person's ability in performing other activities (see table 1). In sum, 22 high-level activities, describing the person's daily life behavior at home, have been considered in the dataset generation. A high-level activity can be composed of atomic actions required to achieve the activity. Considered activities are: eating, dressing, washing, grooming (washing hand/face, hair dry, makeup), toileting, housekeeping, laundry, meal preparation (make coffee, make tea, make sandwich, make hot food, move dish, wash dish, 


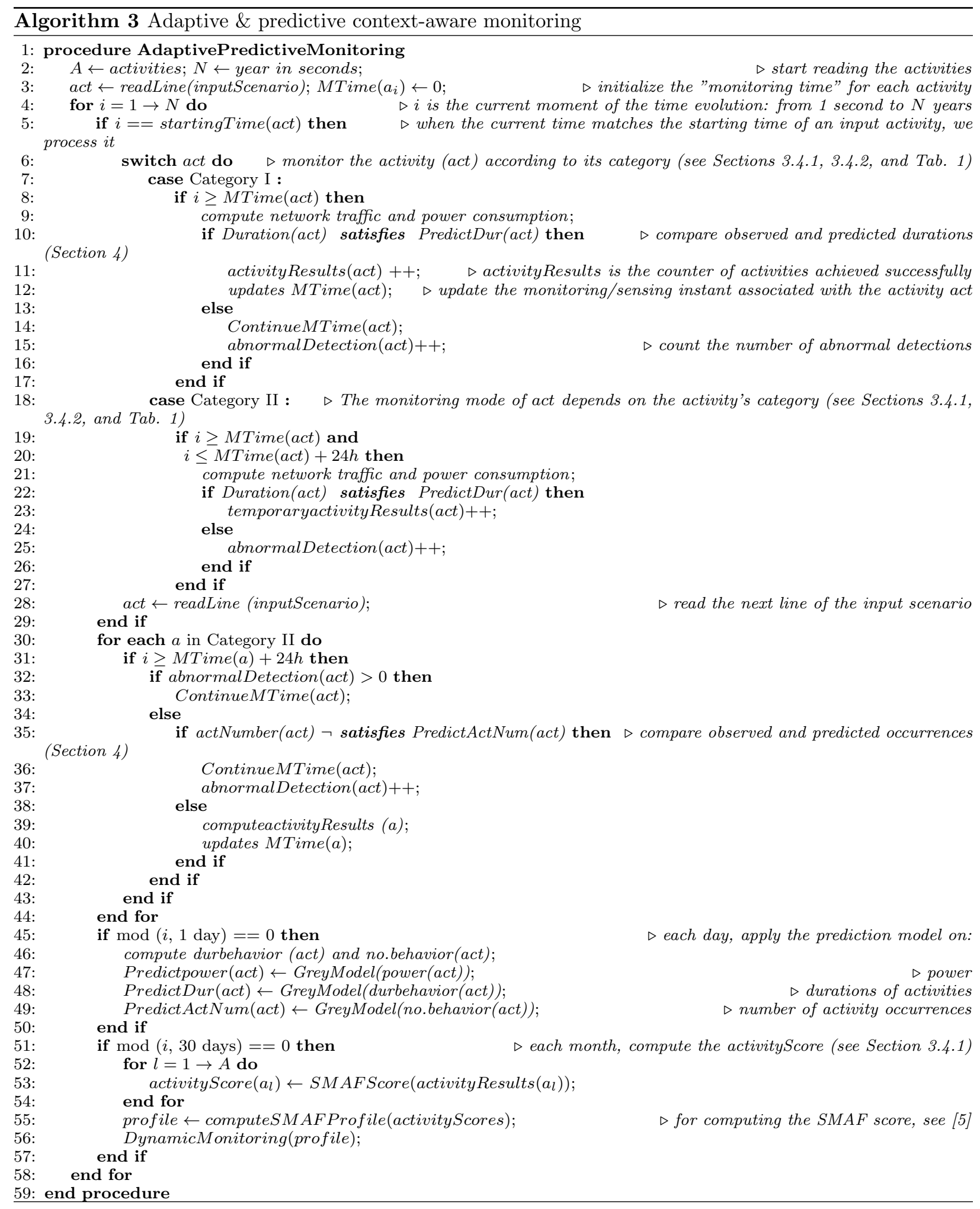

etc.), telephone use, taking medication, walk up/down, walk in/outside, watching TV, reading, and sleeping 
Table 4: Example of sequence of daily activities.

\begin{tabular}{lllll}
\hline Day & Start time & Day & End time & Activity \\
\hline 09 & $08: 10: 39$ & 09 & $08: 30: 32$ & Washing (take shower) \\
09 & $08: 31: 57$ & 09 & $08: 33: 57$ & Drying hair \\
09 & $08: 35: 09$ & 09 & $08: 41: 15$ & Change clothes \\
09 & $08: 42: 21$ & 09 & $08: 51: 05$ & Toileting \\
09 & $08: 52: 55$ & 09 & $08: 57: 34$ & Washing hand/face \\
09 & $08: 58: 55$ & 09 & $09: 06: 39$ & Making coffee \\
09 & $09: 08: 27$ & 09 & $09: 13: 41$ & Washing hand/face \\
09 & $09: 15: 02$ & 09 & $09: 20: 16$ & Making sandwich \\
09 & $09: 21: 40$ & 09 & $09: 45: 00$ & Eating \\
$\ldots$ & $\ldots$ & $\ldots$ & $\ldots$ & $\ldots$ \\
09 & $23: 31: 46$ & 10 & $07: 25: 07$ & Sleeping \\
10 & $07: 27: 59$ & 10 & $07: 35: 00$ & Toileting \\
\hline
\end{tabular}

43. Using the monitoring of one single person with different scenarios, we perform various experimentations and mainly focus on the adaptive monitoring issues. It is worth noting that the proposed framework is not limited to a single person. Indeed, once the actor of a given action or activity is identified (for instance using RFID techniques), the proposed approaches remain unchanged. In our context-aware framework, it is assumed that the considered activities are easily identifiable by relying on the existed infrastructure enriched by the techniques and knowledge of activity recognition [44, 45.

\subsection{Monitoring results}

To show the ability of the adaptive monitoring and the predictive model discussed earlier, we have conducted several simulations for the outcome of the person's behavior in terms of time series during one year. Our experimentations apply the discussed approaches used in Algorithm 3 using two different classes of scenarios. First, we consider scenarios of an elderly having the same level of dependency using the same profile $P_{1}$. $P_{1}$ represents autonomous people or those with the lowest level of dependency. Secondly, we simulate the person's loss of abilities by considering changes in the person's profile and hence in the level of dependency. The considered profile changes are as follows: profile $P_{1}$ during the first 3 months, profile $P_{3}$ from month 4 to 6 , profile $P_{6}$ from month 7 to 9 and finally profile $P_{9}$ for the last 3 months of the year.

The proposed monitoring system aims to reduce data sensing without compromising the reliability of dependency evaluation, forecast the behavior and health status of elderly, and ensure the identification of abnormalities that can be risky for the person. We evaluate the efficiency of our monitoring system using synthetic data generated for long term period with profile changes. These evaluations consider computing process which is related to the number activities that are monitored by the system, the detection of abnormal situations, dependency level, energy and network traffic consumption comparison between the traditional systems (using an unconditional and continuous monitoring) and our adaptive monitoring. To strengthen the flexibility of our evaluation, we avoid rigid and restrictive settings specifically for the network traffic and energy consumption. Therefore, we consider the variation of 3 classes of sensors: high, medium and low nodes used in monitoring of activities (Section 3.4.1). It is obvious that the resources consumption of the system widely depends on the kind of sensors selected to monitor a specific activity. Table 1 gives the classes of different possible sensors used in activities monitoring. For each considered class, we associate a metric function that returns the different consumption values. For example, in the low class, we consider sensors with standard power values: $1 \mu \mathrm{A}, 7.5 \mathrm{~mA}$ and $10.8 \mathrm{~mA}$ for the modes of transmitting, idle (or receiving), and sleeping respectively [46].

\subsubsection{Validation of the adaptive monitoring approach}

We conducted simulations with varying the sensing frequency (i.e., $x$ value) in order to ensure and identify the efficient adjustment of frequencies that combines the optimization of the resources (computing, network, and energy), the dynamic update of the monitoring mode (increase or decrease of $x$ based on health condition), the credibility of dependency evaluation and the guarantee of a high accuracy in the detection of 
Table 5: Resources consumption of the adaptive monitoring (with various frequencies) compared to a continuous monitoring.

\begin{tabular}{ccccc}
\hline$x$ values & Sensing activities (\%) & Power $(\%)$ & Network traffic $(\%)$ & Profile evaluation \\
\hline$X_{1}$ & 60.82 & 66 & 65.05 & $P_{1}$ \\
$X_{2}$ & 54.02 & 55.38 & 54.12 & $P_{1}$ \\
$X_{3}$ & 30.48 & 30.70 & 29.99 & $P_{1}$ \\
$X_{4}$ & 21.49 & 20.96 & 20.40 & $P_{1}$ \\
\hline
\end{tabular}

abnormal and unusual situations whatever the level of the dependency. Identified values are then used with GM $(1,1)$ in order to predict the health conditions based on the previous behavior and the energy used. The first obtained results are presented in Table 5 where we show the percentages for sensing activities, power and network traffic required by our adaptive system when it is compared to the required resources in unconditional continuous monitoring. A variation of the monitoring frequency $x$ is applied on simulated scenarios where the monitored person belongs to the same profile $P_{1}$. With $X_{1}$, all the considered activities (categories: I and II, see Section 3.4.1 have the same $x$ value which refers to a maximum of monitoring which is applied each day. With $X_{2}$, the used $x$ value is set to 1 for Category II, and set to 3 for Category I. Using $X_{3}$, the $x$ value is set to 2 for Category II, and to 5 for Category I. Finally, $X_{4}$ refers to the minimal monitoring hence the $x$ value is set to 3 for Category II and to 10 for Category I. Thanks to this significant optimization, the system succeeds in attaining a perfect accuracy (100\%) for the evaluation of the person's dependency level. Observed results presented in Table 5 are mainly due to the conditional monitoring and its strong link to the person's profile and nature of activities. Hence, extra monitoring and data collection are avoided when it is possible.

By nature, the needs of elderly persons in terms of assistance and services are changing gradually over the time. Therefore, to face the changes of the person's life over long-term the e-health systems must be adaptable. Moreover, it is of paramount importance to guarantee an adequate monitoring that sharps a possible sudden decline regarding the health status. In order to evaluate the adaptability of our approach and test the sensing frequencies, we compare the continuous monitoring and our monitoring system with a set of different $x$. The simulated scenarios consider declines of the person's health status. Specifically, three declines, represented by an ordered profile changes, are used: $P_{1}, P_{3}, P_{6}$ and $P_{9}$. It is worth repeating that the first profile $P_{1}$ represents autonomous persons and that the person's dependency increases when the profile increases. Fig. 4 presents the required amount of resources in terms of computing, network and power and shows the accuracy of the automatic dependency evaluation. In this figure, we compare the continuous monitoring approach with our adaptive monitoring using the different sampling frequencies $X_{i}$ (as detailed previously) and with a user's profile change from the SMAF profile 1 to 9. In Fig. 4.A, we show the number of activities monitored using the continuous and adaptive approaches. In Fig. 4. A and 4 B, we evaluate the resulting power and network consumption for this monitoring.

From Fig. 4 a, we observe that our monitoring system performs a sensing of $61.5 \%$ of activities with $X 1$, $54.3 \%$ with $X 2,35.6 \%$ with $X 3$, and $24.5 \%$ with $X 4$ when compared to a traditional continuous monitoring. With sensing a very low amount of data, we observe a high accuracy related to the decline detection. This is mainly due to the consideration of the person's context. Specifically, the dependency degree and the history of the behavior. Consequently and due to the conditional monitoring that relies only on relevant sensed data, the observed reduction is of $37.2 \%$ for the energy consumption and $38 \%$ of the network traffic with $X 1,48.3 \%$ for the energy and $49.3 \%$ of the network traffic with $X 2,64 \%$ for the energy and $64.6 \%$ of the network traffic with $X 3$, and $74 \%$ for the energy and $74.3 \%$ of the network traffic with $X 4$ (Fig. 4 .b and 4.c).

\subsubsection{Validation of the adaptive monitoring with prediction}

As discussed in Section 4 our proposed predictive model is mainly based on time series analysis. We apply this model to our synthetic dataset in the same way as in the adaptive monitoring approach. In such a way, scenarios consider either the same person's profile or a person with a change of profiles. The sensing frequencies addressed previously are used in the evaluation. The goal of the prediction functionality is to 

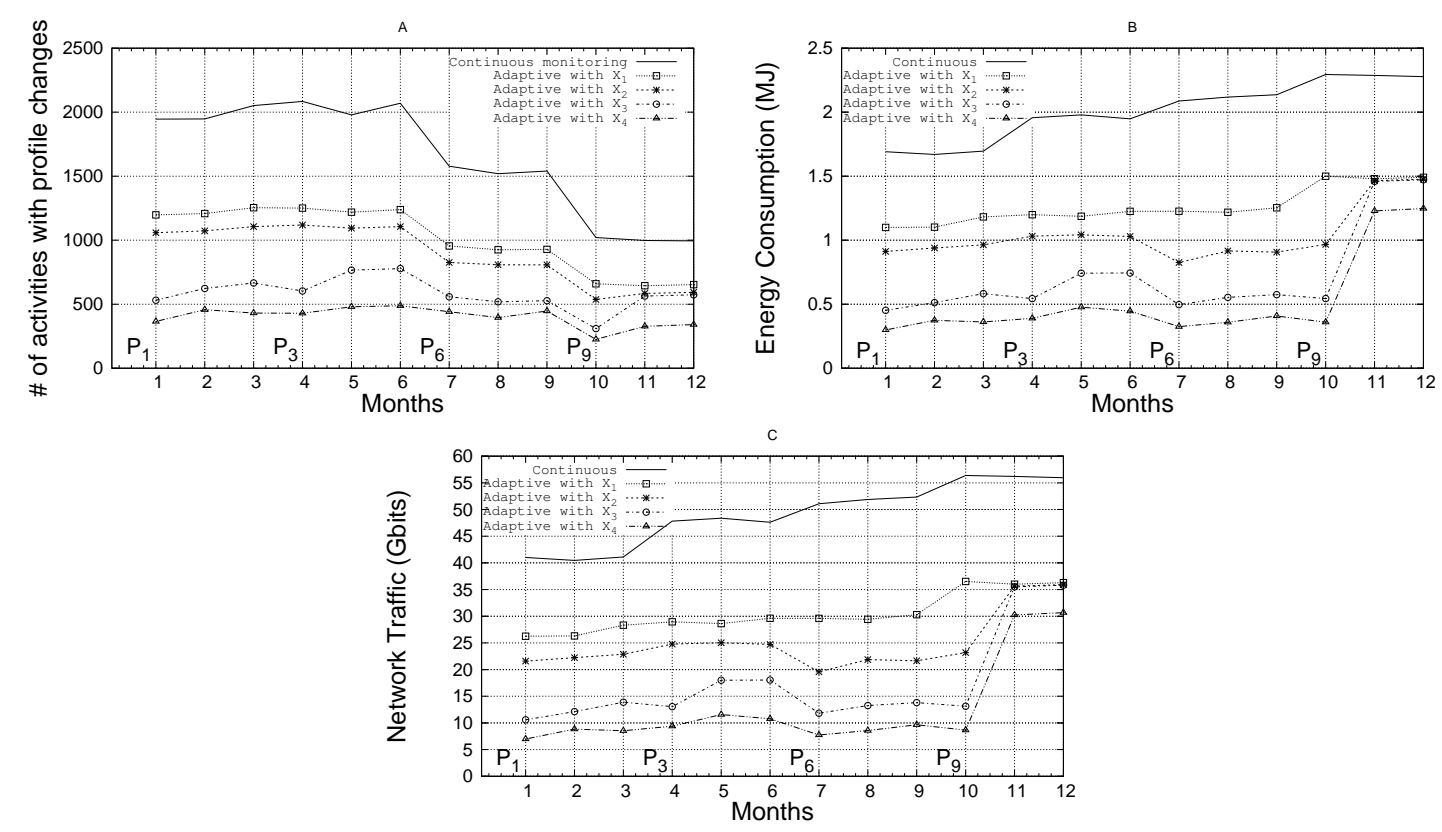

Figure 4: Resources consumption in continuous and adaptive monitoring with health decline. The adaptive monitoring is applied on simulated data, during 12 months, in different modes using $X_{1}, X_{2}, X_{3}$ and $X_{4}$ frequencies. The decline is represented by a change of the SMAF profile of the person as follows: $P_{1}, P_{3}, P_{6}$ then $P_{9}$

Table 6: Adaptive \& predictive monitoring (with various frequencies) compared to a continuous monitoring.

\begin{tabular}{cccccc}
\hline$x$ values & Sensing activities (\%) & Power (\%) & Network traffic (\%) & Abnormal detection (\%) & Profile evaluation \\
\hline$X_{1}$ & 60.82 & 66 & 65.05 & 100 & $P_{1}$ \\
$X_{2}$ & 54.02 & 55.38 & 54.12 & 100 & $P_{1}$ \\
$X_{3}$ & 35.43 & 34.46 & 33.82 & 77.78 & $P_{1}$ \\
$X_{4}$ & 26.22 & 23.93 & 23.43 & 56 & $P_{1}$ \\
\hline
\end{tabular}

provide the system with the ability to detect when a change could occur. Changes are related to how the monitored person performs the daily activities and also how to predict the future behavior based on previous and current situations. GM $(1,1)$ helps to optimize the monitoring system by giving a more accurate map about the person's context. Since it is the context which allows the identification of an abnormal behavior, it is required to have a higher data sensing frequency compared to the adaptive approach. A robust contextaware monitoring system can be evaluated based on how much it can afford a good vision and knowledge of the person's context and how this relevant knowledge is used to timely provide services and assistance. In the context of HSH, it is required to ensure a credible dependency evaluation with a high accuracy for detecting abnormal situations that may represent a risk for the monitored person. The Grey model GM $(1,1)$ allows predicting the evolution of the health conditions based on the daily behavior and the energy consumption required in the monitoring. Hence, the system is constantly aware of the health status of the elderly and its dependency level using minimum data acquired with optimal sensing frequencies. Note that the energy consumption is used mainly because it reflects well the activities of the person.

Experimentation, performed with the Grey model, uses the different $x$ values presented in the adaptive monitoring on different scenarios within the same person's profile (Table 6) and with profile changes (Fig. 6). We compare the continuous monitoring and our adaptive system with the forecasting model. The comparison comprises resources consumption (computing, network, and energy), detection of abnormal situations and dependency level evaluation.

Our prediction model, based on GM $(1,1)$, optimizes the monitoring system by learning the normal daily behavior. It extracts the real deviation of the elderly's behavior compared to the norm. Consequently, 


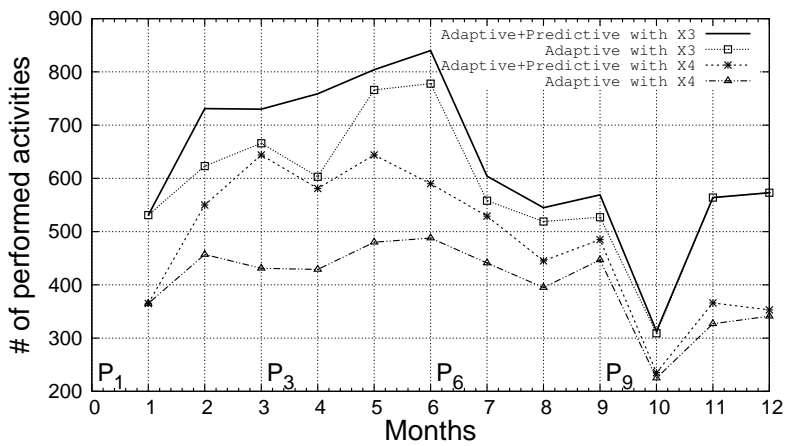

Figure 5: Comparison between the adaptive monitoring without and with prediction in terms of number of monitored activities. We monitor a person with a health condition decline. This decline is represented in simulated data by a change in the dependency profile as follows: $P_{1}, P_{3}, P_{6}$ then $P_{9}$. The adaptive monitoring is achieved using two different sensing frequencies $X_{3}$ and $X_{4}$

predictions are more accurate in understanding the person's context and in detecting the abnormalities of the daily behavior. The abnormal detection with the forecasting model requires a higher frequency of the monitoring and computing that are related to an extra number of activities. These activities are widely related to the nature of the abnormal situation. We can observe this difference by comparing the resources consumption between Table 5 (adaptive monitoring) and Table 6 (predictive monitoring) using the $X 3$ and $X 4$ frequencies. Fig. 5 focuses on the comparison between the adaptive and predictive approaches within different profiles and using the sensing frequencies $X 3$ and $X 4$. Note that the results regarding the adaptive and predictive monitoring are almost the same for $X 1$ and $X 2$ since there is a high frequency in sensing data that allows monitoring all the activities.

Fig. 6] shows the evaluation of the predictive monitoring with different profiles and using different sensing frequencies. The results are similar to those obtained with the adaptive approach. Consequently, even with a decline in the health status, ensuring a timely and context-aware monitoring does not imply a huge amount of data sensing if the monitoring algorithm is efficiently designed. In Fig. 6. a, we observe that the system computes $61.5 \%$ of activities with $X 1,54.3 \%$ with $X 2,38.3 \%$ with $X 3$, and $29.3 \%$ with $X 4$ compared to the continuous monitoring. Hence, an important amount of energy and network bandwidth is saved (Fig. 6. b and 6. c).

It is recalled that an abnormal situation is tied to unusual behaviors in performing the daily activities in terms of duration, repeatability and absence activities. Based on our predictive model, the maximum and minimum values of duration and frequency for each activity are computed as described in section 4. Compared to prior periods, major differences that may occur in the achievement of a given activity, represent a notable change in the person's behavior. In other words, if performing the daily activities exceeds the expected values (forecasting range), the system will identify an abnormal behavior and thus updates the sensing frequency. Sensors are thus forced to continue the monitoring till the behavior becomes as usual. Table 7 give an example of the activities' input values and their forecasted values. The abnormal situations occur in the input scenario with some particular values regarding the performed activities and their nature.

Table 7: Current and forecasting values of activities (duration/number) using the predictive model.

\begin{tabular}{l|ccc|cc|l} 
& \multicolumn{3}{|c|}{ Forecasting values } & \multicolumn{2}{c|}{ Input values } & \\
\hline Activity & Grey value & Min & Max & Duration $(s)$ & Number & Status \\
\hline Meal preparation & 2683 & 2146 & 3219 & 2450 & - & regular \\
Meal preparation & 6 & 4 & 8 & - & 6 & regular \\
Take shower (washing) & 843 & 543 & 1143 & 1199 & - & irregular \\
Toileting & 447 & 358 & 537 & 421 & - & regular \\
Toileting & 5 & 4 & 6 & - & 8 & irregular \\
Sleep & 28127 & 22501 & 33752 & 28366 & - & regular \\
Medication use & 1 & 1 & 1 & - & 0 & irregular \\
\hline
\end{tabular}

Fig. 7 compares the accuracy of detecting the abnormal situations using different sensing frequencies. The $X_{1}$ frequency reflects the highest monitoring and provides a full detection of abnormal situations (a total of 529 cases). The results reveal that our proposed system, enriched with the predictive model of 

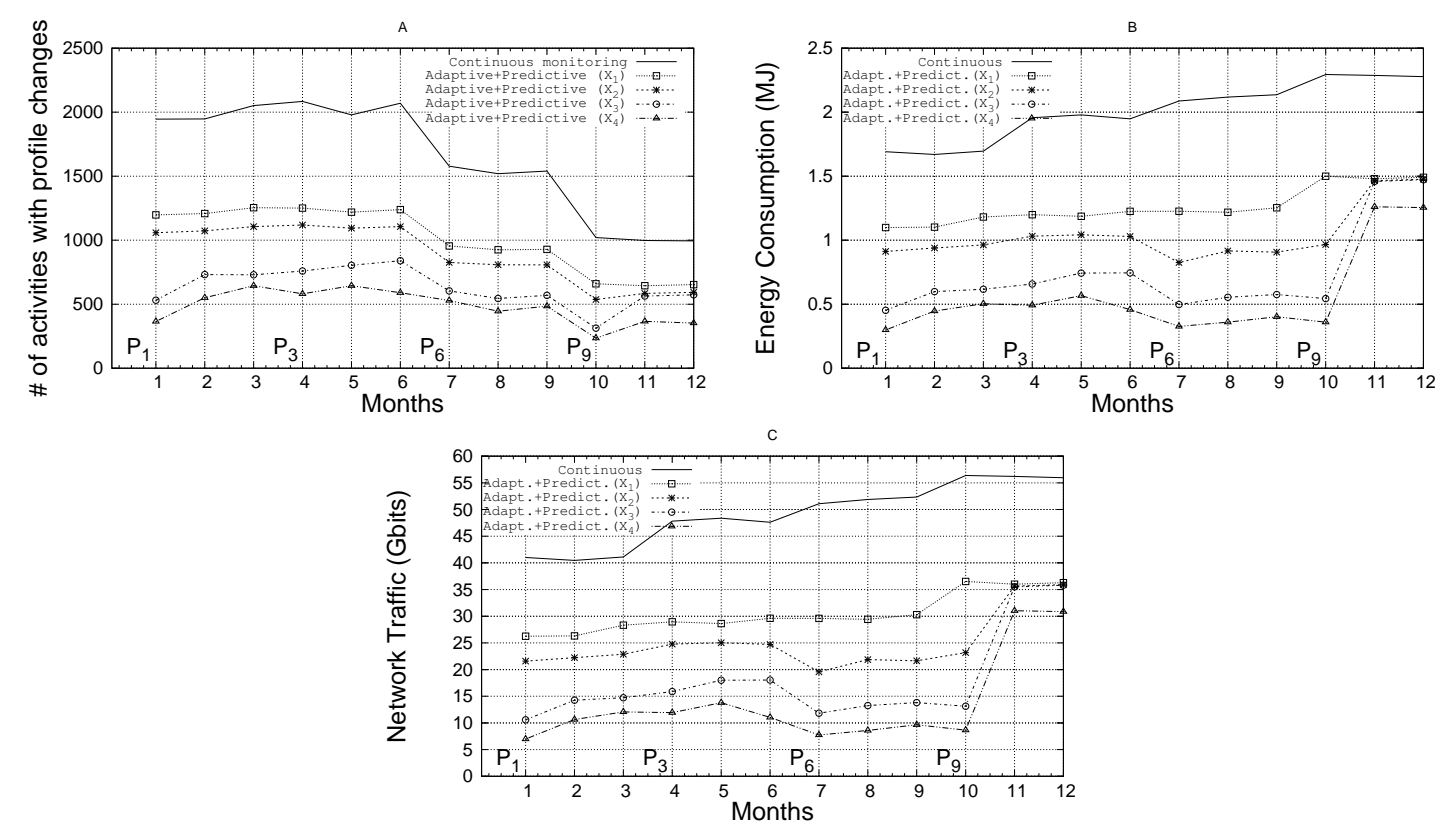

Figure 6: Comparison between the continuous monitoring and the adaptive monitoring with prediction in terms of resources consumption. The resources are the number of processed activities, i.e., computation (A), the energy consumption (B), and the network traffic $(\mathrm{C})$. The prediction is achieved using the GM $(1,1)$-based approach and considers the profile changes of the person from $P_{1}$ to $P_{9}$

the person's behavior, succeed to ensure a high accuracy of the detection with the same sensing frequency. Despite that $X_{2}$ senses $54.3 \%$ of the whole activities, the frequency matches the performance of $X_{1}$ and succeeds to reach a perfect (100\%) detection of abnormal situations. This result is due to the frequencies used in sensing, which are more context-aware. Indeed, the sensing depends on the nature of each activity and the probability that an abnormal situation could occur. For instance, the monitoring of some activities such as meal preparation and washing is always frequent and almost continuous if compared to other activities such as watching $T V$ and reading that are monitored periodically and at low frequency. In the same Fig. 7. we observe that the accuracy of the abnormal behavior detection is $95.8 \%$ with $X_{3}$ and $91.9 \%$ with $X_{4}$ by sensing $38.3 \%$ and $29.3 \%$ of the whole activities respectively. Generally, based on the daily behaviors for elderly, the person who becomes more dependent tends predominantly to perform a less number (and hence durations) of ADL/IADL activities (frequency) and spend a long time (duration) for leisure activities with less mobility. Fig. 8 a gives an example showing the number of meal preparation activities (as one ADL activity) performed by a person who lost his autonomy (declining profiles). Fig. 8 b shows the duration spent in watching $T V$ (as one leisure activity) for the same person during the same period.

There is a strong relationship between the durations required in performing most of the activities, the person's consumed energy, and the energy consumed by the system to monitor the activities. Indeed, the major part of the activities requires energy to be performed correctly, hence if a given activity takes a long time, the person's consumed energy will increase. Similarly, when one activity is achieved several times, the energy consumption of the associated sensors (i.e., the sensors that monitor such activity) increases. In realworld smart spaces, the real-time person's energy consumption is easy to extract and does not require any huge computing or complex sensors. Indeed, an electricity usage monitor can be included in the monitoring system to exactly indicate the consumed energy per device or appliance. Fig. 8 8 c and 8 . d show the durations required to achieve the ADL activities and leisure activities respectively for one year. Based on the previous observations, we can use the consumption of energy (used to monitor ADL and leisure activities) as an interesting indicator to understand the person's behavior and predict future behavioral changes. For this, we classify the energy required for monitoring activities into two main categories. The first category is 


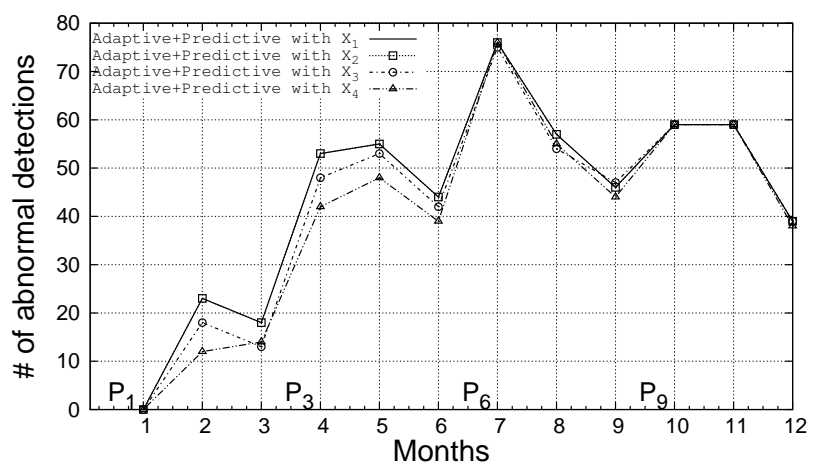

Figure 7: Accuracy detection of abnormalities using an adaptive monitoring with the GM $(1,1)$ prediction. The adaptive monitoring uses the following frequencies: $X_{1}, X_{2}, X_{3}$ and $X_{4}$. The subject's profile evolves in the following order: $P_{1}, P_{3}, P_{6}$ then $P_{9}$
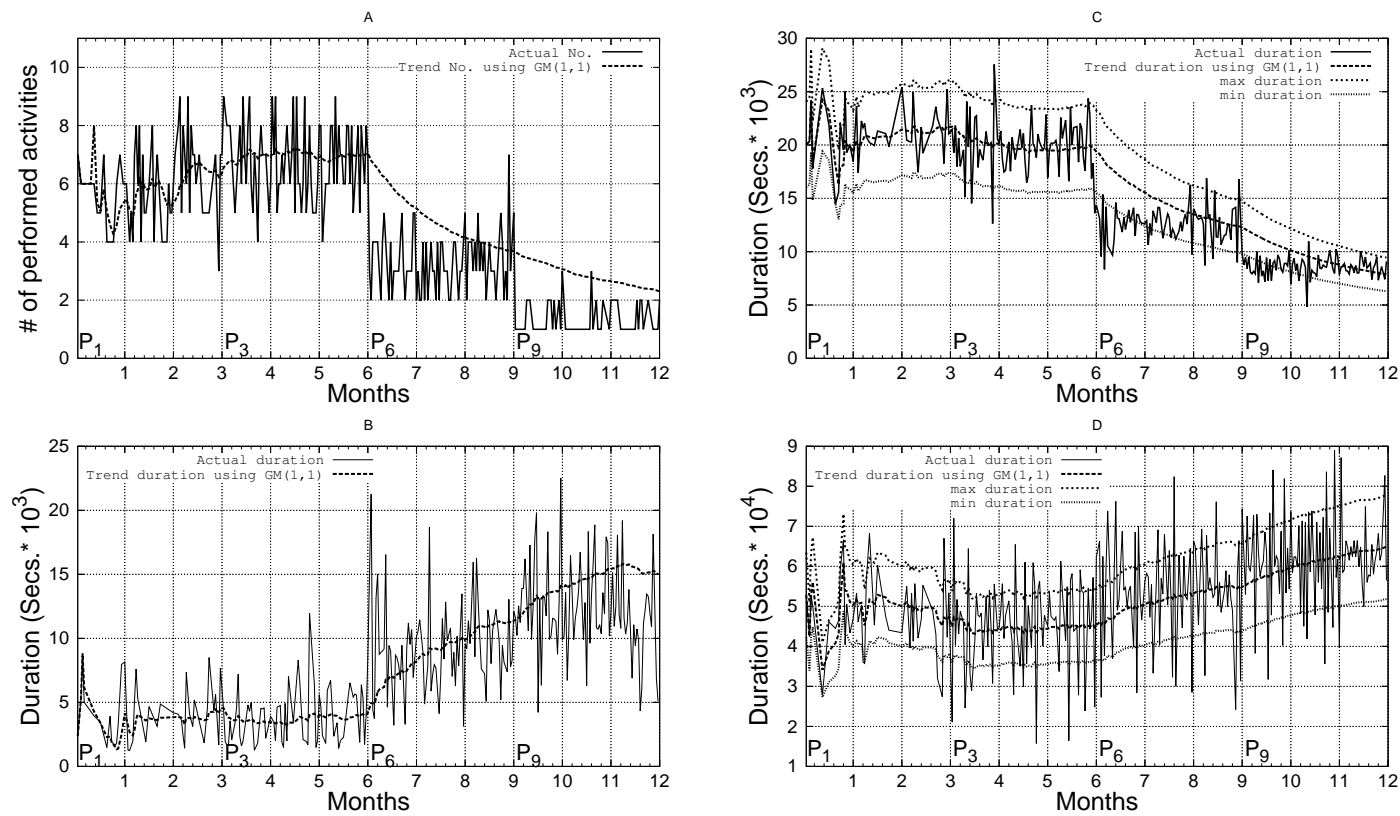

Figure 8: Input values and predicted trend of daily activities depending on the health deterioration. (A) An example of decrease of basic activities when the dependency level increases. The considered activity is meal preparation. (B) An example of duration increase regarding leisure activities when the dependency level increases. The evaluated activity is watching $T V$. (C) input and predicted durations required for basic activities when the dependency level increases. The trend is decreasing for all ADL activities (D) input and predicted durations required for the leisure activities when the dependency level increases. The trend is increasing.

related to ADL and IADL activities. It represents the major part of activities such as meal preparation, eating, washing, etc. The second category is related to leisure activities and represents a minor part if compared to the first category. It covers the energy required for monitoring activities like watching $T V$ and reading.

Fig. 9. a. presents the energy required to monitor the activities for ADL, IADL, and leisure activities during one year. We compare input and predicted values using GM(1,1). We use the energy consumption as an indicator for detecting abnormalities as follows. An abnormality is detected when the following conditions are satisfied. First, when the used energy is less than the predicted values for important activities (i.e., ADL/IADL). Second, when the used energy is more than the predicted values for leisure activities. The 

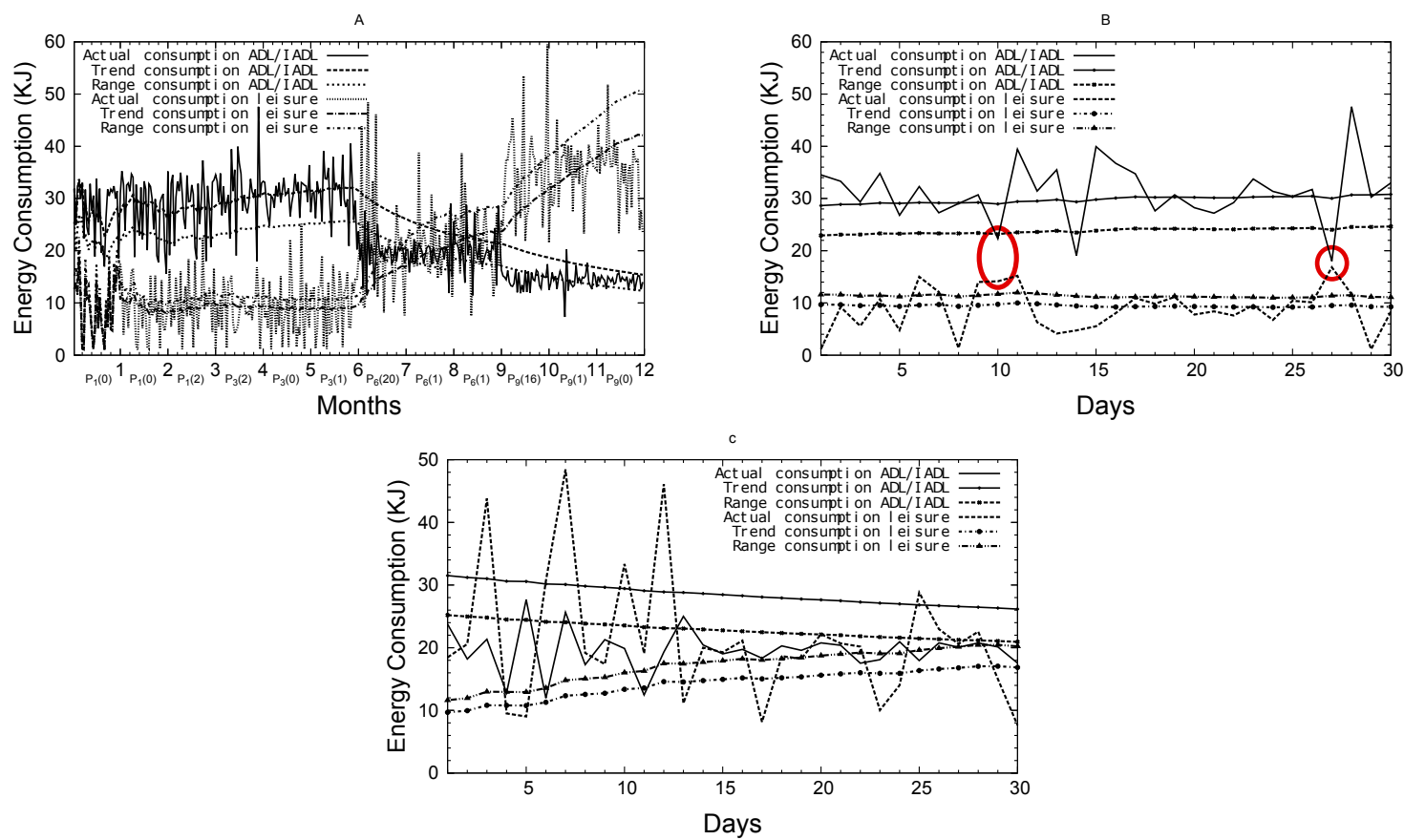

Figure 9: The power consumption as an indicator to understand the behavior and predict its changes. We compare the energy range required in the monitoring of $\mathrm{ADL} / \mathrm{IADL}$ activities and the one required for leisure activities. (A) Observed and predicted energy required to monitor ADL/IADL and leisure activities with profile changes during 12 months. (B) The energy required to monitor ADL/IADL and leisure activities during month 4. (C) The energy required to monitor ADL/IADL and leisure activities during month 7 .

results reveal 20 detected changes during month 7 . Thus, there is a high probability that a significant profile change occurred. We observe also 16 detected changes during the month 10 with no change during the last month. This is explained by a higher use of energy for leisure activities, which makes the second condition unsatisfied. Fig. 9.b and 9.c focus on months 4 and 7 respectively. During month 4, the system detects only 2 cases of abnormal behavior. Consequently, there is a low probability that the usual behavior is affected. However, during month 7, 20 cases of abnormalities were detected, hence, there is a high probability that the usual behavior will change. In Fig. 9a, our energy-based reasoning complies with the input scenario hence this reasoning can be used to predict changes. Indeed, the person's profile is changed from $P_{3}$ (month 6) to $P_{6}$ (month 7) which presents a loss of autonomy. Note that from months 3 to 4 , the profile changed from $P_{1}$ to $P_{3}$ without a loss of autonomy since $P_{1}, P_{2}$ and $P_{3}$ represent autonomous persons (Section 3.1).

\section{Conclusion}

e-health monitoring systems monitor the person's activities and provide helpful feedback particularly when risky situations occur. In this work, we have proposed an adaptive and predictive context-aware monitoring system that tackles some drawbacks of existing e-health solutions, such as continuous monitoring, lack of abnormality detection methods, and prediction techniques requiring long training periods. More specifically, we improved e-health monitoring by maintaining a connection to existing medical standards, such as the models used in geriatrics, and by ensuring applicability in real-world conditions through the optimization of resource usage. The system proposed in this paper is able to evaluate the person's dependency, predict its future health condition, and anticipate its deterioration before major complications. To optimize the use of resources, the system dynamically adapts sensing frequency, by using smart conditional processing that considers the person's profile. The forecasting model embedded in the system is able to predict a person's change in behavior by analyzing a minimum amount of sensed data within a short period of training. To 
evaluate the system, a pseudo variable-length Markovian model was used to generate a long-term realistic dataset that can be used by real-life implementations for testing purposes. The system's predictive approach is highly accurate in detecting abnormal behaviors and unusual situations for all the dependency levels of monitored persons: $100 \%$ of accuracy in high monitoring $\left(X_{2}\right), 95.8 \%$ in medium monitoring $\left(X_{3}\right)$ and $91.9 \%$ with a minimum of monitoring $\left(X_{4}\right)$. Adaptive monitoring applied to different profiles of persons, reduced energy consumption by $48.3 \%$, network traffic by $49.3 \%$ and daily activities processing by $54.3 \%$. This notable accuracy is mainly due to the adaptation of the monitoring to the person's activity (Table 1), the anticipation of behavior changes (Table 6), and the variation of sampling frequencies (Algorithm 3).

Future work includes extending the number of actions considered in our scenario generation in order to refine our knowledge of a person's behavior. Temporal logic between activities (time intervals) such as sequential, concurrent, and interleaved activities should be taken into account and inserted in the dataset. Other parameters could be added for more realistic scenarios such as seasonality, location, gender, age, etc. We could combine several prediction models and evaluate their impact on the anticipation of risks and resources consumption. Finally, we plan to extend the monitoring with vital signs by integrating new constrained environments, such as Body Area Networks (BAN). It is worth noting that our experimental results and analysis were restricted to simulated scenarios and generated datasets. We plan on investigating our system with real-world data, produced by complex infrastructures under real conditions.

\section{References}

[1] U.N., Department of Economic and Social Affairs, http://esa.un.org/unpd/wpp/ (2015).

[2] H. H. Mshali, T. Lemlouma, D. Magoni, Context-aware adaptive framework for e-health monitoring, in: IEEE International Conference on Data Science and Data Intensive Systems, 2015, pp. 276-283.

[3] R. Heeks, Health information systems: Failure, success and improvisation, International Journal of Medical Informatics 75 (2) (2006) 125-137.

[4] B. Kaplan, K. D. Harris-Salamone, Health it success and failure: Recommendations from literature and an amia workshop, Journal of the American Medical Informatics Association 16 (3) (2009) 291-299.

[5] H. Mshali, T. Lemlouma, D. Magoni, Analysis of dependency evaluation models for ehealth services, in: IEEE Global Communications Conference, 2014, pp. 2429-2435.

[6] N. Nguyen, D. Phung, S. Venkatesh, H. Bui, Learning and detecting activities from movement trajectories using the hierarchical hidden markov model, in: IEEE Conference on Computer Vision and Pattern Recognition, Vol. 2, 2005, pp. 955-960.

[7] D. Trabelsi, S. Mohammed, F. Chamroukhi, L. Oukhellou, Y. Amirat, An unsupervised approach for automatic activity recognition based on hidden markov model regression, IEEE Trans. Autom. Sci. Eng. 10 (3) (2013) 829-835.

[8] L. Atallah, G.-Z. Yang, The use of pervasive sensing for behaviour profiling - a survey, Pervasive and Mobile Computing 5 (5) (2009) 447-464.

[9] M. Mao, E. Chirwa, Application of grey model gm $(1,1)$ to vehicle fatality risk estimation, Technological Forecasting and Social Change 73 (5) (2006) 588-605.

[10] H. Mshali, T. Lemlouma, D. Magoni, A predictive approach for efficient e-health monitoring, in: IEEE International Conference on e-Health Networking, Applications and Services, 2015, pp. 268-273.

[11] R. Jouini, T. Lemlouma, K. Maalaoui, L. A. Saidane, Employing grey model forecasting gm $(1,1)$ to historical medical sensor data towards system preventive in smart home e-health for elderly person, in: International Wireless Communications and Mobile Computing Conference, 2016, pp. 1086-1091.

[12] V. Osmani, S. Balasubramaniam, D. Botvich, A bayesian network and rule-base approach towards activity inference, in: Vehicular Technology Conference, 2007, pp. 254-258.

[13] T. Nguyen, S. Gupta, S. Venkatesh, D. Phung, A bayesian nonparametric framework for activity recognition using accelerometer data, in: International Conference on Pattern Recognition, 2014, pp. 2017-2022.

[14] N. Suryadevara, S. Mukhopadhyay, R. Wang, R. Rayudu, Forecasting the behavior of an elderly using wireless sensors data in a smart home, Eng. Appl. Aartif. Intel. 26 (10) (2013) 2641-2652.

[15] A. R. M. Forkan, I. Khalil, Z. Tari, S. Foufou, A. Bouras, A context-aware approach for long-term behavioural change detection and abnormality prediction in ambient assisted living, Pattern Recognition 48 (3) (2015) 628-641.

[16] H. Zheng, H. Wang, N. Black, Human activity detection in smart home environment with self-adaptive neural networks, in: IEEE International Conference on Networking, Sensing and Control, 2008, pp. 1505-1510.

[17] H. Li, Q. Zhang, P. Duan, A novel one-pass neural network approach for activities recognition in intelligent environments, in: World Congress on Intelligent Control and Automation, IEEE, 2008, pp. 50-54.

[18] S. Chatterjee, Q. Xie, K. Dutta, A predictive modeling engine using neural networks: Diabetes management from sensor and activity data, in: International Conference on e-Health Networking, Applications and Services, 2012, pp. $230-237$.

[19] H.-H. Hsu, C.-C. Chen, Rfid-based human behavior modeling and anomaly detection for elderly care, Mob. Inf. Syst. 6 (4) (2010) 341-354 
[20] B. Yuan, J. Herbert, Context-aware hybrid reasoning framework for pervasive healthcare, Pers. Ubiquit. Comput. 18 (4) (2014) 865-881.

[21] R. Kadouche, H. Pigot, B. Abdulrazak, S. Giroux, User's behavior classification model for smart houses occupant prediction, in: Activity Recognition in Pervasive Intelligent Environments, Springer, 2011, pp. 149-164.

[22] F. Paganelli, D. Giuli, An ontology-based system for context-aware and configurable services to support home-based continuous care, IEEE Transactions on Information Technology in Biomedicine 15 (2) (2011) $324-333$.

[23] A. König, C. F. Crispim-Junior, A. Derreumaux, G. Bensadoun, P.-D. Petit, F. Bremond, R. David, F. Verhey, P. Aalten, P. Robert, Validation of an Automatic Video Monitoring System for the Detection of Instrumental Activities of Daily Living in Dementia Patients, J Alzheimers Dis. 44 (2) (2015) 675-685.

[24] T. G. Stavropoulos, G. Meditskos, I. Kompatsiaris, Demaware2: Integrating sensors, multimedia and semantic analysis for the ambient care of dementia, Pervasive and Mobile Computing 34 (Supplement C) (2017) 126 - 145.

[25] D. Riboni, C. Bettini, Cosar: hybrid reasoning for context-aware activity recognition, Personal and Ubiquitous Computing 15 (3) (2011) 271-289.

[26] B. Reeder, J. Chung, T. Le, H. Thompson, G. Demiris, Assessing older adults' perceptions of sensor data and designing visual displays for ambient assisted living environments: An exploratory study, Methods Inf. Med. 53 (3) (2014) 152.

[27] F. Cardinaux, S. Brownsell, D. Bradley, M. S. Hawley, A home daily activity simulation model for the evaluation of lifestyle monitoring systems, Comput. Biol. Med. 43 (10) (2013) 1428-1436.

[28] J. W. Lee, S. Cho, S. Liu, K. Cho, S. Helal, Persim 3d: Context-driven simulation and modeling of human activities in smart spaces, IEEE Trans. Autom. Sci. Eng. 12 (4) (2015) 1243-1256.

[29] J. Qi, P. Yang, M. Hanneghan, S. Tang, Multiple density maps information fusion for effectively assessing intensity pattern of lifelogging physical activity, Neurocomputing 220 (2017) 199-209.

[30] N. Noury, T. Hadidi, Computer simulation of the activity of the elderly person living independently in a health smart home, Computer Methods and Programs in Biomedicine 108 (3) (2012) 1216-1228.

[31] H. Medjahed, D. Istrate, J. Boudy, B. Dorizzi, Human activities of daily living recognition using fuzzy logic for elderly home monitoring, in: IEEE International Conference on Fuzzy Systems, 2009, pp. 2001-2006.

[32] M. Raiche, R. Hébert, M.-F. Dubois, N. R. Gueye, N. Dubuc, Yearly transitions of disability profiles in older people living at home, Archives of Gerontology and Geriatrics 55 (2) (2012) 399-405.

[33] T. Lemlouma, S. Laborie, P. Roose, Toward a context-aware and automatic evaluation of elderly dependency in smart homes and cities, in: IEEE International Symposium on a World of Wireless Mobile and Multimedia Networks, 2013, pp. $1-6$.

[34] P. Augustyniak, Request-driven ecg interpretation based on individual data validity periods, in: IEEE International Conference on Engineering in Medicine and Biology, 2007, pp. 3777-3780.

[35] T. V. Kasteren, Datasets for activity recognition, http://sites.google.com/site/tim0306 [Accessed: June 2017] (2010).

[36] F. J. Ordóñez, P. de Toledo, A. Sanchis, Activity recognition using hybrid generative/discriminative models on home environments using binary sensors, Sensors 13 (5) (2013) 5460-5477.

[37] B. Logan, J. Healey, M. Philipose, E. M. Tapia, S. Intille, A long-term evaluation of sensing modalities for activity recognition, in: International conference on Ubiquitous computing, 2007, pp. 483-500.

[38] D. J. Cook, A. S. Crandall, B. L. Thomas, N. C. Krishnan, Casas: A smart home in a box, IEEE Computer 46 (7) (2013) $62-69$.

[39] G. Virone, B. Lefebvre, N. Noury, J. Demongeo, Modeling and computer simulation of physiological rhythms and behaviors at home for data fusion programs in a telecare system, in: International Workshop on Enterprise Networking and Computing in Healthcare Industry, 2003, pp. 111-117.

[40] B. Kormányos, B. Pataki, Multilevel simulation of daily activities: Why and how?, in: IEEE International Conference on Computational Intelligence and Virtual Environments for Measurement Systems and Applications, 2013, pp. 1-6.

[41] H. Mshali, T. Lemlouma, D. Magoni, Context-aware adaptive framework for e-health monitoring, in: IEEE International Conference on Green Computing and Communications, 2015, pp. 276-283.

[42] A. Galata, N. Johnson, D. Hogg, Learning variable-length markov models of behavior, Computer Vision and Image Understanding 81 (3) (2001) 398-413.

[43] H. Mshali, T. Lemlouma, D. Magoni, eHealth Monitoring Open Data Project, https://sourceforge.net/projects/ ehealthmonitoringproject/ (2015).

[44] S. Chernbumroong, S. Cang, A. Atkins, H. Yu, Elderly activities recognition and classification for applications in assisted living, Expert Syst. Appl. 40 (5) (2013) 1662-1674.

[45] E. M. Tapia, S. S. Intille, K. Larson, Activity recognition in the home using simple and ubiquitous sensors, in: International Conference on Pervasive Computing, 2004, pp. 158-175.

[46] TR1000, ASH transceiver data sheet, RF Monolithics Inc., http://www.datasheetlib.com/ [Accessed: June 2017] (2017). 